\title{
Design of Ferromagnetic Shape Memory Alloy Composites
}

\author{
MASAHIRO KUSAKA* AND MinORU TAYA \\ Department of Mechanical Engineering \\ Center for Intelligent Materials and Systems \\ University of Washington \\ Box 352600, Seattle, WA, 98195-2600, USA
}

(Received April 17, 2003)

(Revised September 12, 2003)

\begin{abstract}
Ferromagnetic shape memory alloy (FSMA) composites composed of a ferromagnetic material and a shape memory alloy (SMA) are key material systems for fast-responsive and compact actuators.

The function of ferromagnetic material is to induce magnetic force which is then used to induce the stress in the SMA, resulting in the stress-induced martensite transformation (SIM), i.e. change in the Young's modulus, stiff (austenite) to soft (martensite). This SIM-induced phase change causes larger deformation in the SMA, which is often termed as "superelastic".

This paper discusses a simple model by which the stress and strain field in the FSMA composites subjected to bending and torsion loading are computed with the aim of identifying the optimum geometry of FSMA composites. The results of the present analytical study are utilized to design torque actuator (bending of FSMA composite plate) and spring actuator (torsion of helical FSMA composite spring).
\end{abstract}

KEY WORDS: shape memory alloy, ferromagnetic material, stress-induced martensite transformation, superelasticity, bending plate, coil spring.

\section{INTRODUCTION}

$\mathbf{R}$ ECENTLY, FERROMAGNETIC SHAPE memory alloys (FSMAs) attract strong attention as a fast responsive actuator material. There are three actuation mechanisms identified in FSMAs, (1) magnetic field-reduced phase transformation, (2) martensite variant rearrangement and (3) hybrid mechanism by magnetic field gradient [1,2]. The first mechanism often requires large magnetic field, thus necessitating the design of large electromagnetic driving unit, not suited for compact actuators, while the second mechanism can provide large strain but at low stress level. Therefore, use of the hybrid mechanism is the most effective to design high-force actuators, yet at fast speed.

\footnotetext{
*Author to whom correspondence should be addressed. E-mail: tayam@u.washington.edu 
The hybrid mechanism is a sequence of chain reaction events, applied magnetic field gradient, magnetic force in a FSMA, stress-induced phase transformation from stiff austenite to softer martensite, resulting in a large deformation, yet large stress can be realized due to superelastic plateau in the stress-strain curve of a FSMA. In the hybrid mechanism, the magnetic force $F$ is given by

$$
F=\mu_{0} V M \frac{\partial H}{\partial x}
$$

where $\mu_{0}$ is the magnetic permeability in vacuum, $V$ is the volume of a ferromagnetic material, $M$ is the magnetization vector and $H$ is the magnetic field, thus $F$ is proportional to both magnetization vector and magnetic field gradient. It is noted that the magnetic force $F$ influences the internal stress field within a FSMA, i.e., the larger $F$ is, the larger stress field is induced in FSMA. It is also here that use of a portable electromagnet or permanent magnet can provide large magnetic field gradient, resulting in larger magnetic force, thus larger stress-induced martensite phase transformation.

The cost of processing FSMAs such as FePd [2] is usually very expensive. Superelastic shape memory alloys (SMAs) have high mechanical performances, large transformation strain and stress capability. But, the speed of superelastic SMAs by changing temperature is usually slow. If a FSMA composite composed of a ferromagnetic material and a superelastic SMA can be developed, cost-effective and high-speed actuators can be designed. In the design of this composite, the requirements are: no plastic deformation of the ferromagnetic material and large transformation strain in superelastic SMA. It is necessary to design the optimum microstructure (cross-section) of composite with high performance (high load capacity and large deformability) while satisfying these requirements. In order to obtain the optimum microstructure of FSMA composites with high performance, one needs to use either numerical models such as finite element method (FEM), or analytical model. There have been a number of works on finite element analysis (FEA) of SMA structures [3-7]. The FEA which uses commercial FEM is time consuming in the preliminary design. It would be easier for a designer to use a simple analytical model to obtain the optimal microstructure of FSMA composite, if the simple analytical model provides closed form solutions. We made a preliminary model for FSMA composites [8]. The analytical model in this paper is a further extension of our preliminary model, and it is aimed at detailed modeling of the superelastic behavior of a SMA in a FSMA composite.

In this study, two cases of loading, bending and the twist modes of the composites are considered with emphasis on how the geometry and the mechanical properties of the components influence the superelastic SMA behavior of the composite. First, the bending deformation of the composite plate with application to torque actuators [9] is theoretically analyzed. That is, the relation between the curvature and the bending moment for the composite plate. Next, the spring of the composite wire with the rectangular section form is designed in consideration of application to spring actuators [10], and the deformation characteristic of the spring is examined. For both models of bending and torsion of FSMA composites, the optimized microstructures of the composites are identified with the aim of maximizing force and deformation of FSMA composite actuators. 


\section{SUPERELASTIC BEHAVIOR OF BENDING COMPOSITE PLATE}

\section{Analytical Model}

For bending type actuation, the laminated composite plate composed of a ferromagnetic material layer and superelastic SMA layer as shown in Figure 1(a), is examined. The composite plate is subject to bending moment $M$ induced by the magnetic force generated by the ferromagnetic material. After the maximum bending stresses on the plate surface of SMA layer reach the transformation stress (onset of superelastic plateau in the upper loop of the stress-strain curve, Figure 2(b)), the phase transformation proceeds from the plate surface as shown in Figure 1(b). The stress in the transformed region remains constant due to the superelastic behavior of SMA. It is assumed throughout in this paper to facilitate the analysis that the superelastic loop of SMA is "flat" i.e. no working-hardening type slope allowed, and the Young's modulus of the austenite is the same as that of the martensite. These assumptions allow us to obtain simple closed form solutions in the present model, although the predictions are still to the first order approximation. The aim of using this simple model is to identify the best thickness ratio of a ferromagnetic layer and SMA layer in the composite plate.

Then, the relation between the bending moment and the curvature is theoretically calculated by using stress-strain curves of the constituent materials. Figure 2(a) shows the

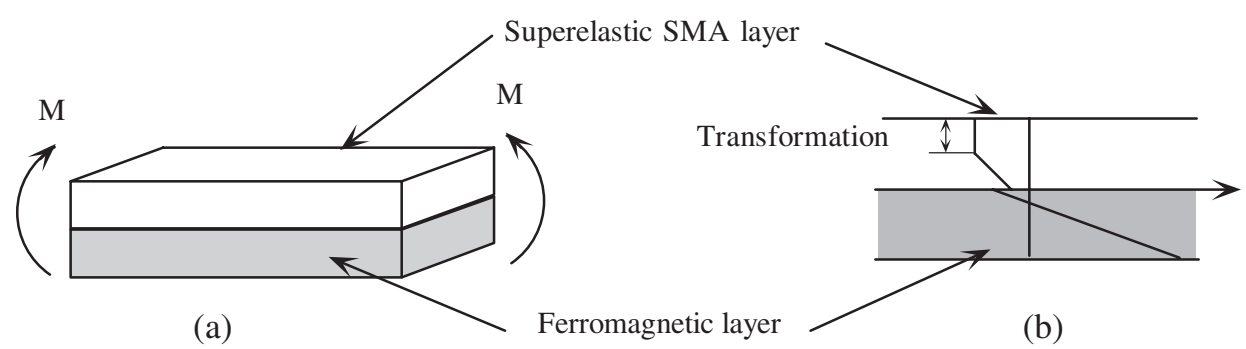

Figure 1. Composite plate for bending mode actuation: (a) material composition; (b) stress distribution in cross section.

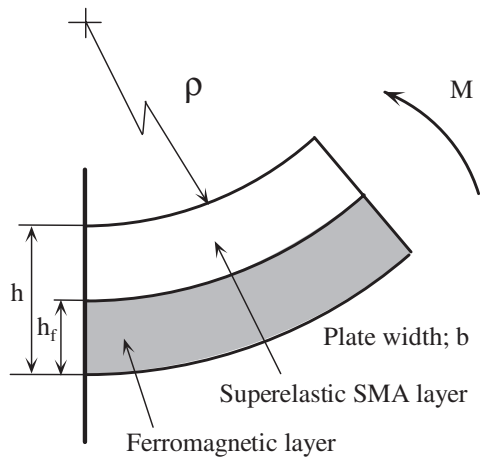

(a)

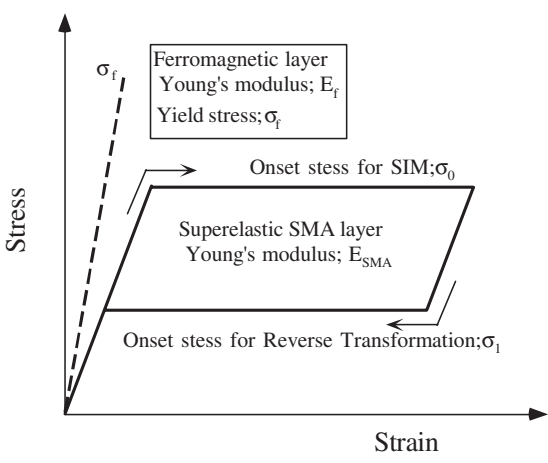

(b)

Figure 2. Material properties and model for the theoretical examination: (a) plate bending model; (b) stressstrain relations for ferromagnetic material and superelastic SMA. 
analytical model. Radius of curvature of the composite plate subject to bending moment $M$ is $\rho$, the thickness of the composite plate is $h$, the thickness of the ferromagnetic layer is $h_{\mathrm{f}}$, and the plate width is $b$. Figure 2(b) shows the stress-strain curves of the ferromagnetic material and the superelastic SMA, where the Young's modulus of the ferromagnetic material is $E_{\mathrm{f}}$, that of the SMA are $E_{\mathrm{SMA}}$, the yield stress of the ferromagnetic material is $\sigma_{\mathrm{f}}$, and only elastic portion of the ferromagnetic material is shown. The onset stress for phase transformation of superelastic SMA is $\sigma_{0}$, the onset stress for reverse transformation is $\sigma_{1}$ in the superelastic loop portion of SMA. As a result, the relation between the bending moment and the curvature of the composite plate also is expected to exhibit the superelastic loop if properly designed. This superelastic loop of the FSMA composites is indeed desired.

The curvature which reaches yield stress $\sigma_{\mathrm{f}}$ in a ferromagnetic layer and the curvature which reaches transformation stress $\sigma_{0}$ in superelastic SMA layer are strongly influenced by the mechanical properties and the thickness of both materials. Stress distribution is classified into the following three cases because of the relation between the transformation stress in the SMA layer and the yield stress of a ferromagnetic layer.

Case 1 The stress in a ferromagnetic layer reaches the yield stress $\sigma_{\mathrm{f}}$, before reaching the transformation stress $\sigma_{0}$ in the superelastic SMA layer.

The stress distribution of this case upon loading and unloading is shown in Figure 3, where the bending stress by elastic deformation is illustrated in each material.

Case 2 The stress in a ferromagnetic layer reaches the yield stress, after SMA layer reaching the transformation stress in some part.

The stress distribution of Case 2 upon loading and unloading is shown in Figure 4. Under increasing bending moment first elastic stress distribution (a), then the stress in the SMA layer reaches the transformation stress $\sigma_{0}$ at the position of $y_{1}(\mathrm{~b})$, and when the transformation domain advances to $y_{1}=Y_{1}$, a ferromagnetic layer reaches the yield stress $\sigma_{\mathrm{f}}(\mathrm{c})$. It is noted in (b)-(e) that $Y_{1}$ remains constant until $y_{3}$ reaches $Y_{1}$. During unloading, the stress decreases first elastically in all domains (d), next, the stress becomes constant from the upper part of the SMA layer to the position of $y_{3}$ where the stress reached reverse transformation stress $\sigma_{1}(\mathrm{e})$. In addition, after the stress at location $y_{3}=Y_{1}$ reaches $\sigma_{1}$, the stress inside portion $\left(y<y_{2}\right)$ decreases elastically $(f)$. Finally, the stress in the entire SMA layer decreases elastically when the stress in the SMA on the top surface becomes small than $\sigma_{1}(\mathrm{~g})$.

Case 3 The stress in a ferromagnetic layer reaches the yield stress, after the entire domain of the superelastic SMA layer reaching the transformation stress $\sigma_{0}$.



Figure 3. Changes in stress distribution in cross section according to load (Case 1). 

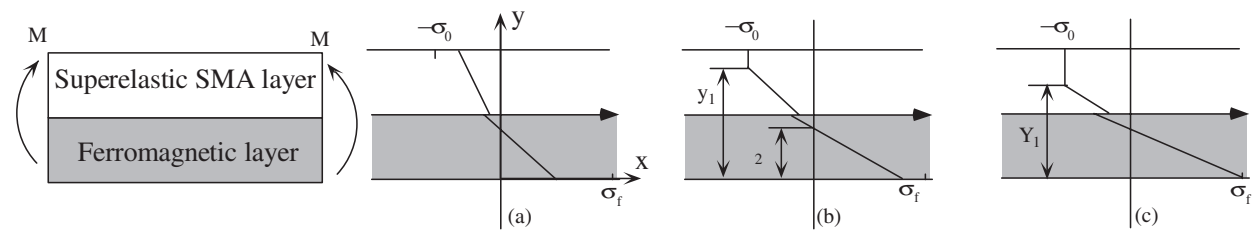

Loading
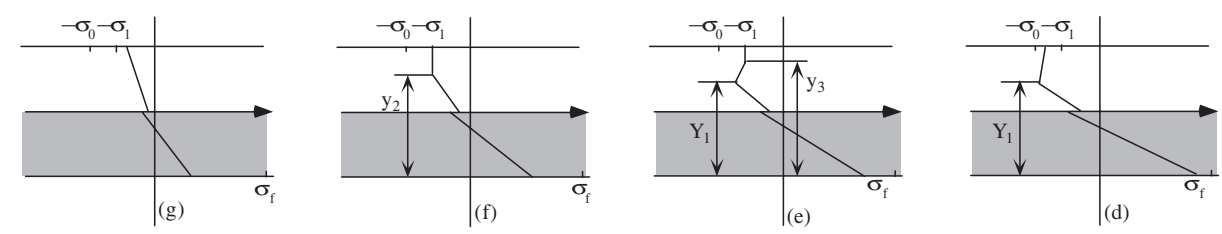

Unloading

Figure 4. Changes in stress distribution in cross section according to load (Case 2).
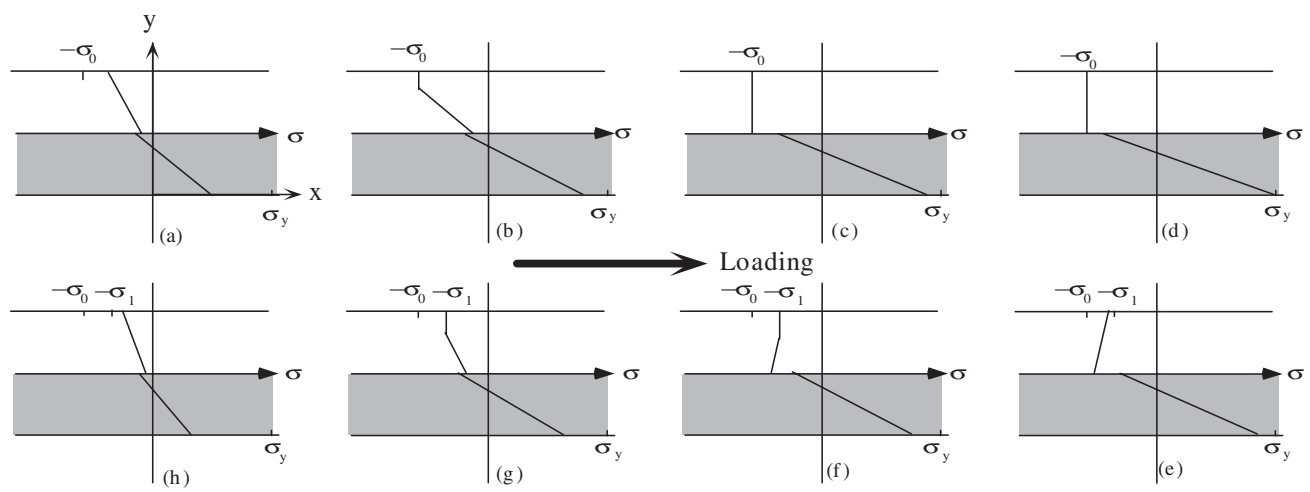

Unloading

Figure 5. Changes in stress distribution in cross section according to load (Case 3).

The stress distribution of Case 3 upon the loading and the unloading is shown in Figure 5. In early stage of loading, the stress in a ferromagnetic layer does not reach the yield stress yet even after the stress in all domains of the SMA layer reaches the transformation stress $\sigma_{0}$ (c). A neutral axis position changes with an increase in the load, and the stress reaches the yield stress $\sigma_{\mathrm{f}}$ finally in a ferromagnetic layer (d). The process of unloading is shown in Figure $5(\mathrm{e})-(\mathrm{h})$.

For each stress distribution $\sigma_{x}(y)$ of the three cases, the following equations are valid, i.e. the equilibrium of force and moment.

$$
\begin{gathered}
\int_{0}^{h} \sigma_{x}(y) b d y=0 \\
M=-\int_{0}^{h} \sigma_{x}(y) y b d y
\end{gathered}
$$


The neutral axis position and the relation between the moment and the curvature are obtained by solving these equations. Let us focus on the case 2, particularly the stress state of Figure 4(b). When a neutral axis position is $\xi_{2}$, and the transformation stress position is $y_{1}$, the stress distribution in each domain becomes

in ferromagnetic layer $\left(0<y<h_{\mathrm{f}}\right)$

$$
\sigma(y)=E_{\mathrm{f}} \frac{\xi_{2}-y}{\rho}
$$

in SMA layer below the transformation stress $\sigma_{0}\left(h_{\mathrm{f}}<y<y_{1}\right)$

$$
\sigma(y)=E_{\mathrm{SMA}} \frac{\xi_{2}-y}{\rho}
$$

in the transformation domain of SAM $\left(y_{1}<y<h\right)$

$$
\sigma(y)=-\sigma_{0}
$$

By substituting Equations (3), (4), and (5) to Equations (2), unknown $\xi_{2}$ and $y_{1}$ are solved and they are given by

$$
\begin{aligned}
& \frac{\xi_{2}}{h}=-\left\{\left(\frac{E_{\mathrm{f}}}{E_{\mathrm{SMA}}}-1\right) \frac{h_{\mathrm{f}}}{h}+\frac{\sigma_{0}}{E_{\mathrm{SMA}}} \frac{\rho}{h}\right\} \\
&+ \sqrt{\frac{E_{\mathrm{f}}}{E_{\mathrm{SMA}}}\left(\frac{E_{\mathrm{f}}}{E_{\mathrm{SMA}}}-1\right)\left(\frac{h_{\mathrm{f}}}{h}\right)^{2}+2 \frac{\sigma_{0}}{E_{\mathrm{SMA}}} \frac{\rho}{h}\left\{1+\left(\frac{E_{\mathrm{f}}}{E_{\mathrm{SMA}}}-1\right) \frac{h_{\mathrm{f}}}{h}\right\}} \\
& \frac{y_{1}}{h}=\frac{\xi_{2}}{h}+\frac{\sigma_{0}}{E_{\mathrm{SMA}}} \frac{\rho}{h}
\end{aligned}
$$

Moreover, by substituting Equations (3)-(7) to Equation (2b), the relation between the normalized bending moment and curvature is obtained as

$$
\begin{array}{r}
\frac{M}{E_{\mathrm{SMA}} b h^{2}}=\frac{h}{\rho}\left[\frac{E_{\mathrm{f}}}{E_{\mathrm{SMA}}}\left\{\frac{1}{3}\left(\frac{h_{\mathrm{f}}}{h}\right)^{3}-\frac{1}{2} \frac{\xi_{2}}{h}\left(\frac{h_{\mathrm{f}}}{h}\right)^{2}\right\}+\frac{1}{3}\left\{\left(\frac{y_{1}}{h}\right)^{3}-\left(\frac{h_{\mathrm{f}}}{h}\right)^{3}\right\}\right. \\
\left.-\frac{1}{2} \frac{\xi_{2}}{h}\left\{\left(\frac{y_{1}}{h}\right)^{2}-\left(\frac{h_{\mathrm{f}}}{h}\right)^{2}\right\}\right]+\frac{1}{2} \frac{\sigma_{0}}{E_{\mathrm{SMA}}}\left\{1-\left(\frac{y_{1}}{h}\right)^{2}\right\}
\end{array}
$$

Equation (8) is valid for the range of curvature, i.e. from the curvature with transformation stress $\sigma_{0}$ in top $(y=h)$ of SMA layer to the curvature with yield stress $\sigma_{\mathrm{f}}$ at bottom $(y=0)$ of ferromagnetic layer. This range of the curvature is given by

$$
\frac{\sigma_{0}}{E_{\mathrm{SMA}}} \frac{2\left\{1+\left(\left(E_{\mathrm{f}} / E_{\mathrm{SMA}}\right)-1\right)\left(h_{\mathrm{f}} / h\right)\right\}}{1+\left(\left(E_{\mathrm{f}} / E_{\mathrm{SMA}}\right)-1\right)\left(2-\left(h_{\mathrm{f}} / h\right)\right)\left(h_{\mathrm{f}} / h\right)}<\frac{h}{\rho} \leq \frac{h}{\rho_{1}}
$$


where

$$
\begin{aligned}
\frac{h}{\rho_{1}}= & \left\{\left(\frac{\sigma_{\mathrm{f}}}{E_{\mathrm{f}}}+\frac{\sigma_{0}}{E_{\mathrm{SMA}}}\right)^{2}\right\} /\left\{-\left\{\frac{\sigma_{\mathrm{f}}}{E_{\mathrm{f}}}\left(\frac{E_{\mathrm{f}}}{E_{\mathrm{SMA}}}-1\right) \frac{h_{\mathrm{f}}}{h}-\frac{\sigma_{0}}{E_{\mathrm{SMA}}}\right\}\right. \\
& \left.+\sqrt{\left\{\frac{\sigma_{\mathrm{f}}}{E_{\mathrm{f}}}\left(\frac{E_{\mathrm{f}}}{E_{\mathrm{SMA}}}-1\right) \frac{h_{\mathrm{f}}}{h}-\frac{\sigma_{0}}{E_{\mathrm{SMA}}}\right\}^{2}+\left(\frac{\sigma_{\mathrm{f}}}{E_{\mathrm{f}}}+\frac{\sigma_{0}}{E_{\mathrm{SMA}}}\right)^{2}\left(\frac{E_{\mathrm{f}}}{E_{\mathrm{SMA}}}-1\right)\left(\frac{h_{\mathrm{f}}}{h}\right)^{2}}\right\}
\end{aligned}
$$

Similarly, the relations between the bending moment and the curvature for the three cases of Figures 3-5 can be calculated. The results for Cases 1, 2 and 3 are shown in Appendix. The conditions under which three cases are valid, are obtained as

Case 1

$$
\frac{\sigma_{\mathrm{f}}}{\sigma_{0}}<\frac{E_{\mathrm{f}}}{E_{\mathrm{SMA}}} \frac{1+\left(\left(E_{\mathrm{f}} / E_{\mathrm{SMA}}\right)-1\right)\left(h_{\mathrm{f}} / h\right)^{2}}{1+\left(\left(E_{\mathrm{f}} / E_{\mathrm{SMA}}\right)-1\right)\left(2-\left(h_{\mathrm{f}} / h\right)\right)\left(h_{\mathrm{f}} / h\right)}
$$

Case 2

$$
2 \frac{h}{h_{\mathrm{f}}}\left\{\frac{\sigma_{0}}{E_{\mathrm{SMA}}}+\frac{\sigma_{0}}{E_{\mathrm{f}}}\left(\frac{h_{\mathrm{f}}}{h}-1\right)\right\}>\frac{h}{\rho_{1}}
$$

Case 3

$$
2 \frac{h}{h_{\mathrm{f}}}\left\{\frac{\sigma_{0}}{E_{\mathrm{SMA}}}+\frac{\sigma_{0}}{E_{\mathrm{f}}}\left(\frac{h_{\mathrm{f}}}{h}-1\right)\right\} \leq \frac{h}{\rho_{1}}
$$

The maximum normalized curvatures in these cases are given by

Case 1

Case 2 Case 3

$$
\frac{h}{\rho}=\frac{\sigma_{\mathrm{f}}}{E_{\mathrm{f}}} \frac{2\left\{1+\left(\left(E_{\mathrm{f}} / E_{\mathrm{SMA}}\right)-1\right)\left(h_{\mathrm{f}} / h\right)\right\}}{1+\left(\left(E_{\mathrm{f}} / E_{\mathrm{SMA}}\right)-1\right)\left(h_{\mathrm{f}} / h\right)^{2}}, \quad \frac{h}{\rho}=\frac{h}{\rho_{1}}, \quad \frac{h}{\rho}=2 \frac{h}{h_{\mathrm{f}}}\left\{\frac{\sigma_{\mathrm{f}}}{E_{\mathrm{f}}}-\frac{\sigma_{0}}{E_{\mathrm{f}}}\left(\frac{h}{h_{\mathrm{f}}}-1\right)\right\}
$$

The maximum deformability of the composite plate can be analyzed for a given set of the mechanical properties and the thickness ratio of materials by using Equation (14).

\section{Analytical Results and Discussion}

The relation between the bending moment and the curvature is predicted by the present model for two types of the composite, i.e. Fe/CuAlMn and $\mathrm{FeCoV} / \mathrm{CuAlMn}$. Figure 6(a) is the idealized stress-strain curves of $\mathrm{Fe}$ and $\mathrm{CuAlMn}$. The results of the predicted relation between the normalized bending moment and the normalized curvature for thickness ratio $h_{\mathrm{f}} / h=0.5$ are shown in Figure $6(\mathrm{~b})$. The state of the stress for this case corresponds to Case 1, Figure 3, i.e. the stress in SMA layer is not superelastic plateau, and thus, the superelastic loop is not observed as evidenced in Figure 6(b). Therefore, the 

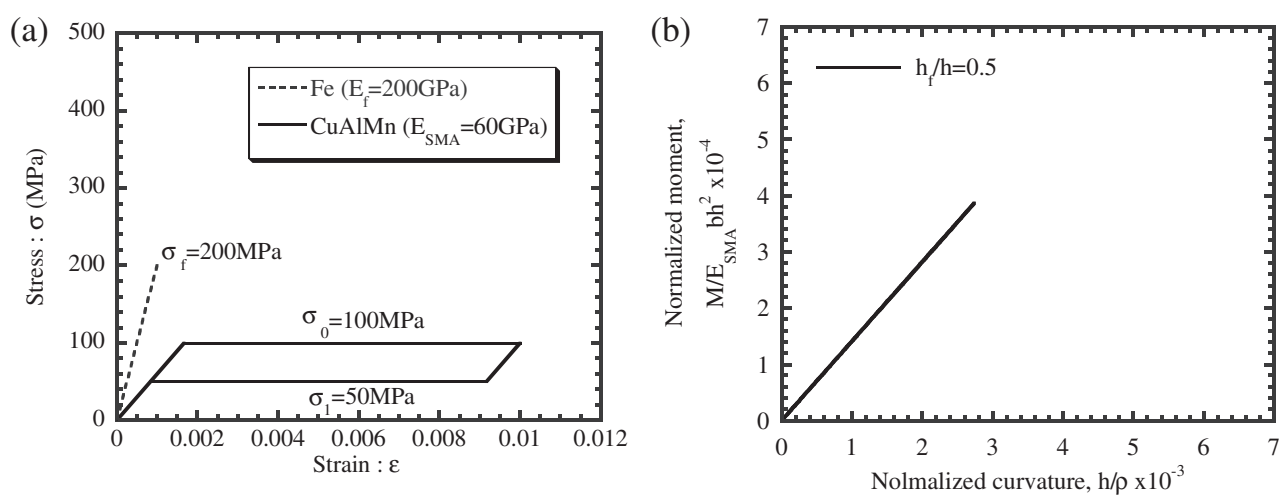

Figure 6. (a) Stress-strain curve for Fe and CuAIMn; (b) relation between normalized bending moment and normalized curvature.
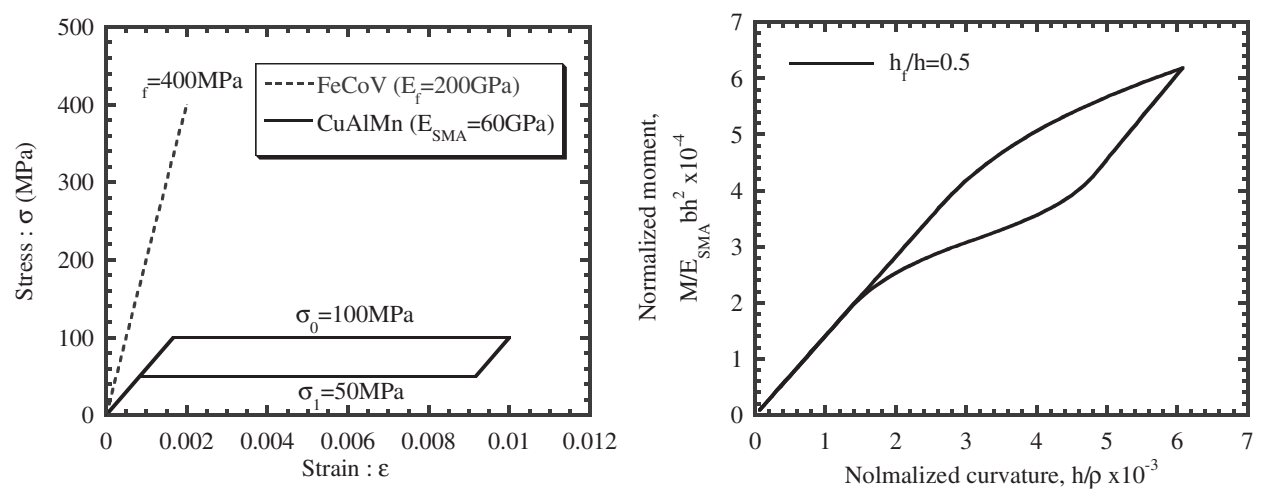

Figure 7. (a) Stress-strain curve for FeCoV and CuAIMn; (b) relation between normalized bending moment and normalized curvature.

composite plate of $\mathrm{Fe}$ and $\mathrm{CuAlMn}$ is undesirable as effective bending actuator component.

Next, the $\mathrm{FeCoV} / \mathrm{CuAlMn}$ composite plate was analyzed by using the mechanical property data shown in Figure 7(a). Figure 7(b) shows the analytical results for $h_{\mathrm{f}} / h=0.5$, exhibiting clearly superelastic behavior. By using FeCoV whose yield stress is larger than $\mathrm{Fe}$, yet its soft magnetic property is better than Fe, we can achieve now the state where most of the CuAlMn layer becomes a transformation domain, corresponding to almost the state of Case 3. Moreover, the maximum curvature was 2.22 times larger and the bending moment was 1.60 times larger than those of the composite with Fe. Therefore, the FSMA composite so identified is promising as an effective bending actuator component.

Next, we performed a set of parametric studies to examine the effects of material parameters $\left(\sigma_{\mathrm{f}}, E_{\mathrm{f}}, \sigma_{0}, \sigma_{1}, E_{\mathrm{SMA}}\right)$ and geometrical parameter, i.e., thickness ratio $\left(h_{\mathrm{f}} / h\right)$. The predicted results are shown in Figure 8, where (a)-(f) denote the case of changing parameters, yield stress of ferromagnetic material $\left(\sigma_{\mathrm{f}}\right)$, the upper plateau stress $\left(\sigma_{0}\right)$ and lower plateau stress $\left(\sigma_{1}\right)$ of $\mathrm{CuAlMn}$ superelastic loop, and ratio of ferromagnetic plate $\left(h_{\mathrm{f}}\right)$ to the composite $(h), h_{\mathrm{f}} / h$, Young's modulus of ferromagnetic material $\left(E_{\mathrm{f}}\right)$ and that of SMA ( $\left.E_{\mathrm{SMA}}\right)$, respectively. 


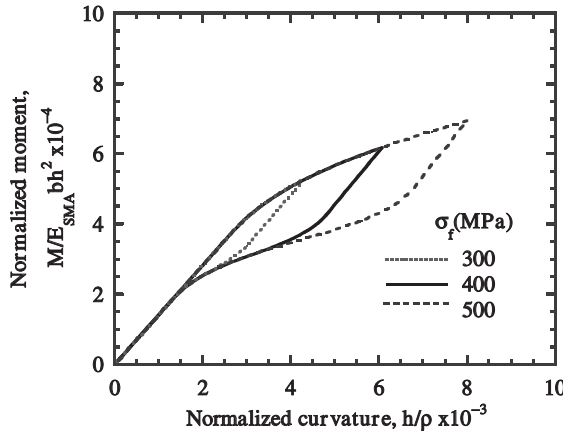

(a)

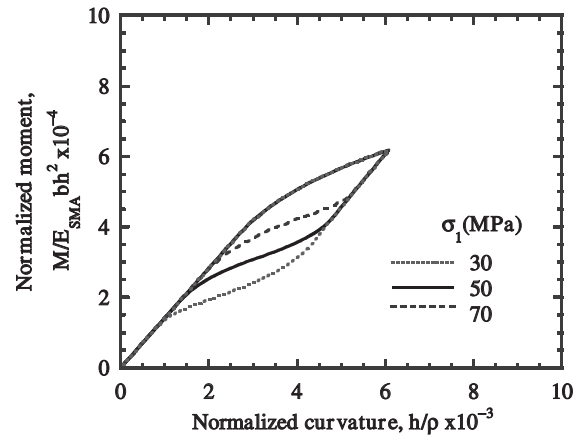

(c)

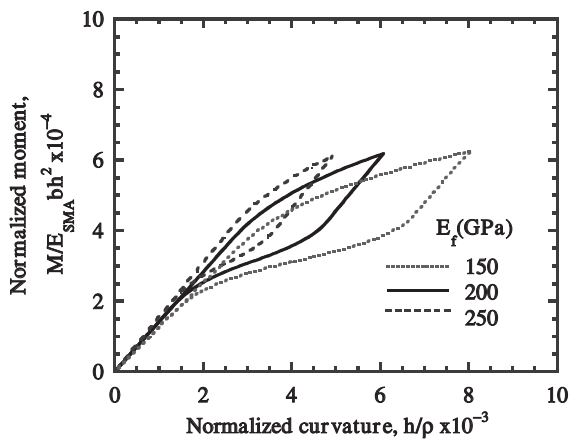

(e)

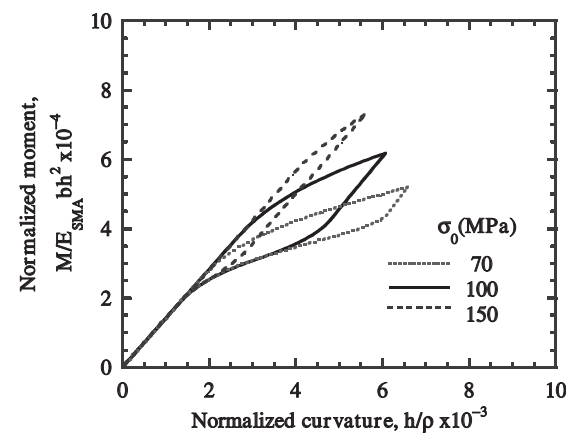

(b)

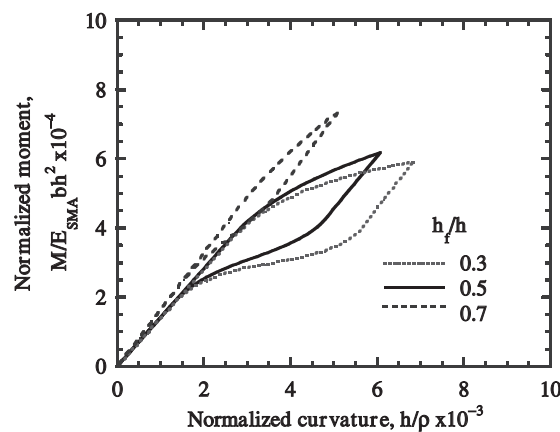

(d)



(f)

Figure 8. Change in superelastic behavior of bending plate influenced by various parameters: (a) yield stress of Fe; (b) upper transformation stress of SMA; (c) lower transformation stress of SMA; (d) thickness ratio of Fe to FSMA composite; (e) Young's modulus of Fe; and (f) Young's modulus of SMA. (a) $\sigma_{f}$; (b) $\sigma_{0}$; (c) $\sigma_{1}$; (d) $h_{f} / h$; (e) $E_{f}$; (f) $E_{S M A}$.

When the yield stress of the ferromagnetic material increases, it is clear from Figure 8(a) that both bending moment and the curvature increase. When transformation stress $\sigma_{0}$ of SMA increases, it is found from Figure 8(b) that the bending moment increases and the curvature decreases. It can be seen from Figure 8(c), the lower limit of the superelastic loop decreases if the reverse transformation stress $\sigma_{1}$ decreases. When the thickness of the ferromagnetic layer increases, it is clear from Figure 8(d) that the bending moment increases 
though the curvature decreases. Oppositely, because the thickness of superelastic SMA layer increases when the thickness of a ferromagnetic layer decreases, the superelasticity behavior increases. Therefore, the bending moment decreases, and the curvature increases. From Figure 8(e), the maximum curvature decreases though the bending moment does not change when the Young's modulus of the ferromagnetic material increases. Therefore, an increase in the Young's modulus of the ferromagnetic material is undesirable as the composite. From Figure 8(f), the bending moment decreases when the Young's modulus of SMA increases. The design of a more high performance FSMA composites becomes possible by the materials design based on the above analysis.

\section{SUPERELASTIC BEHAVIOR OF COIL SPRING MODE OF A COMPOSITE WIRE WITH RECTANGULAR CROSS SECTION}

\section{Analytical Model}

With the aim of designing a high-speed linear actuator, the superelastic characteristic of a coiled spring of the ferromagnetic shape memory composite wire with rectangular section is analyzed. Figure 9 shows the analytical model. The magnetic force is generated in the ferromagnetic material by the magnetic field gradient, and displacement is generated in the spring by the hybrid mechanism described in Introduction. The relation between this spring force and displacement is analyzed.

When axial force $P$ is given to the spring, the wire of the ferromagnetic shape memory composite is subjected to torque $T$. The relation between spring force $P$ and torque $T$ is given by

$$
T=P R \cos \alpha
$$

For a twist angle per unit length of the rectangular section wire of $\omega$, the total twist angle $\phi$ is $2 n \pi R \omega \sec \alpha$ as the total length of the wire is $2 n \pi R \omega \sec \alpha$. Therefore, the displacement of the spring is calculated by the next equation.

$$
\begin{aligned}
\delta & =\delta_{\text {torsion }}+\delta_{\text {shear }} \cong \delta_{\text {torsion }} \\
& =R \phi=2 n \pi R^{2} \omega \sec \alpha
\end{aligned}
$$

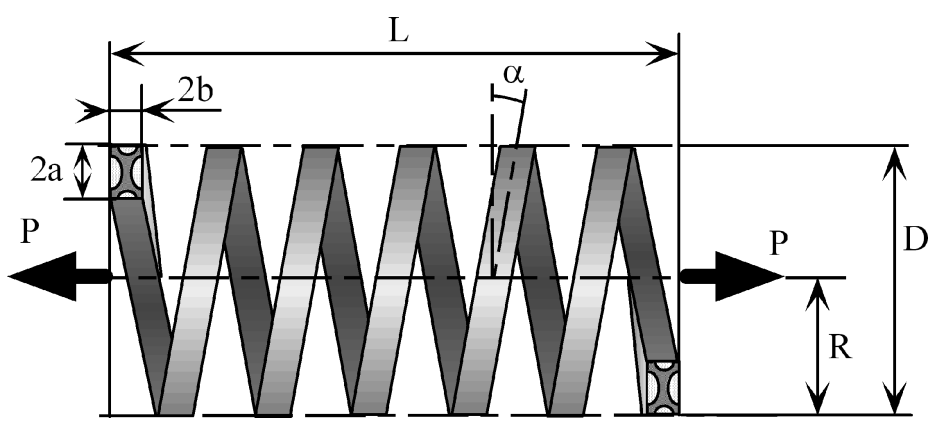

Figure 9. Analytical model of coil spring with rectangular cross section. $D$ : the diameter of spring $(D=2 R)$, $d$ : the diameter of wire, $p$ : the pitch of one cycle, $n$ : the number of turns, $L:$ the length of spring without load $(L=n p), \alpha$ : the inclined angle of the wire to the $x-y$ plane. 
It is assumed in the present model that the displacement due to direct shear, $\delta_{\text {shear }}$ is neglected. This is justified for large ratio of $D$ to $a$ or $b$. Then, the relation between the spring force, $P$ and displacement, $\delta$ can be calculated if the relation between the twist angle per unit length $\omega$ and the torque $T$ of the rectangular section wire is known, which will be obtained in the following.

\section{Analytical Model for Torsion of Composite Wire with Rectangular Section}

To generate large magnetic force by the hybrid mechanism, it is necessary to increase the area of a ferromagnetic material in the rectangular section, while meeting the requirement that the ferromagnetic material should not reach its yield stress. The stress field in the rectangular section can be calculated from the shear strain distribution of the rectangular section for a given twist angle.

Let us look at the rectangular section of a composite with width $2 a$ and height $2 b$ as shown in Figure 9. We introduce the assumption that the spring deformation is uniform along the wire direction ( $z$-axis) and plane displacements $u$ and $v$ are in proportion to $z$, as follows;

$$
u=-\omega y z, \quad v=\omega x z, \quad w=\omega \varphi j(x, y)
$$

where the function $\varphi(x, y)$ is the Saint-Venant's function [11] that satisfies the equilibrium equation and 2D compatibility equation of strain. For the spring with rectangular cross section, the shear strain components are expressed as

$$
\begin{aligned}
& \frac{\gamma_{z x}}{\omega a}=-\frac{16}{\pi^{2}} \sum_{n=1}^{\infty} \frac{(-1)^{n-1}}{(2 n-1)^{2}} \frac{\sinh [(2 n-1) \pi y / 2 a]}{\cosh [(2 n-1) \pi b / 2 a]} \cos [(2 n-1) \pi x / 2 a] \\
& \frac{\gamma_{z y}}{\omega a}=\frac{16}{\pi^{2}} \sum_{n=1}^{\infty} \frac{(-1)^{n-1}}{(2 n-1)^{2}}\left\{1-\frac{\cosh [(2 n-1) \pi y / 2 a]}{\cosh [(2 n-1) \pi b / 2 a]}\right\} \sin [(2 n-1) \pi x / 2 a]
\end{aligned}
$$

Therefore, the effective shear strain acting on the rectangular cross section, $\gamma$ is calculated by

$$
\gamma=\sqrt{\gamma_{z x}^{2}+\gamma_{z y}^{2}}
$$

For $a=2$ and $b=1$, the contour line distributions of shear strain component $\gamma_{z x}, \gamma_{z y}$ and effective shear strain $\gamma$ divided by $a \omega$ are shown in Figure 10(a), (b) and (c) respectively. $\gamma_{z x}$ becomes 0 at $x=-a$ and $a$, and it reaches to the minimum value at $y=b$ on the $y$ axis, and becomes the maximum at $y=-b$ on the $y$ axis. $\gamma_{z y}$ reaches to the minimum value at $x=-2, y=0$, and becomes the maximum at $x=2, y=0$. The normalized effective shear strain, $\gamma / a \omega$ reaches the maximum value 0.930 at the center of long side edges, and reduces toward the center.

The effective shear stress induced in the ferromagnetic material is calculated by multiplying $\gamma$ by the shear modulus $G_{\mathrm{f}}$ of the ferromagnetic material. The effective shear stress distribution of the ferromagnetic material in the rectangular section is calculated for 


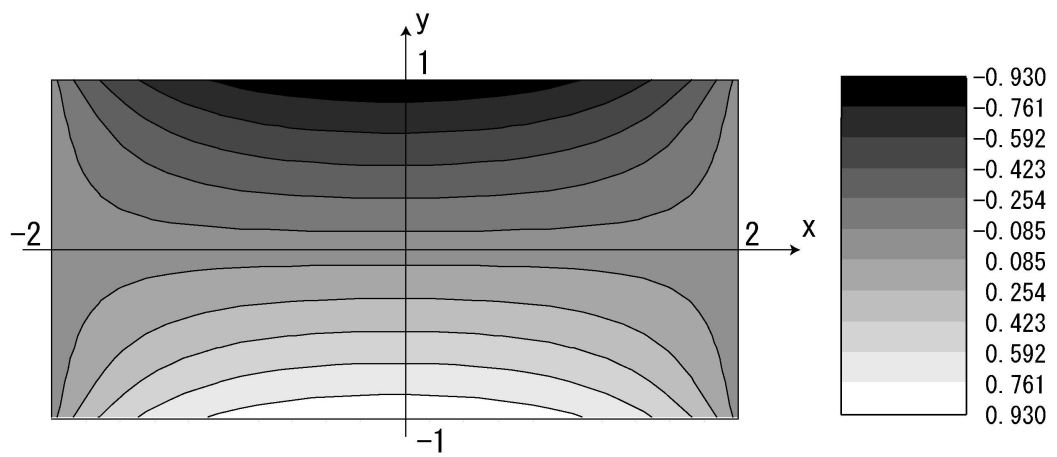

(a)

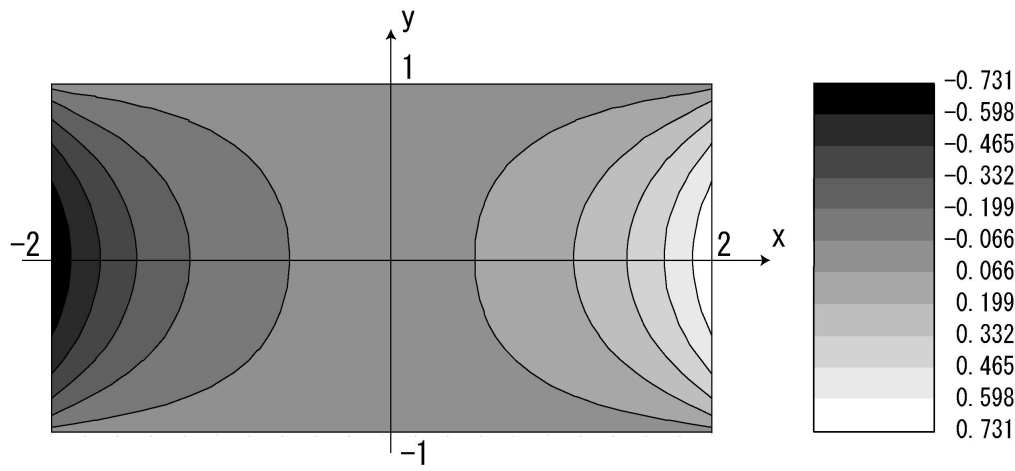

(b)

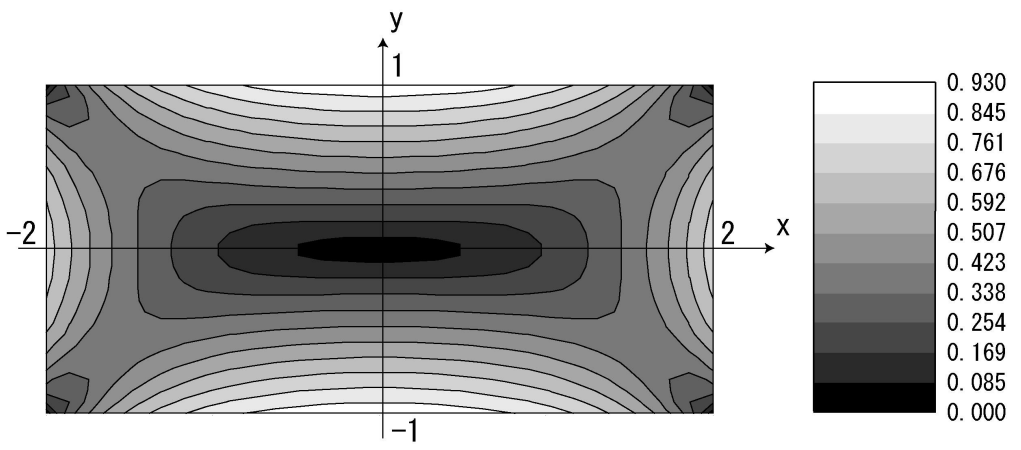

(c)

Figure 10. Contour line distributions of shear strain in rectangular section: (a) $\gamma_{z x} / a \omega$; (b) $\gamma_{z y} / a \omega$; and (c) $\gamma / a \omega$, where $a$ is the length of longer side of a rectangular cross section of a FSMA composite and $\omega$ is the twist angle per unit length.

a given set of twist angle per unit length $\omega$, size $a$ and $b$. Then, the optimum shape of the ferromagnetic material can be determined from its domain under the condition that the effective shear stress does not exceed the yield stress in shear $\tau_{\mathrm{f}}$ of the ferromagnetic material. 


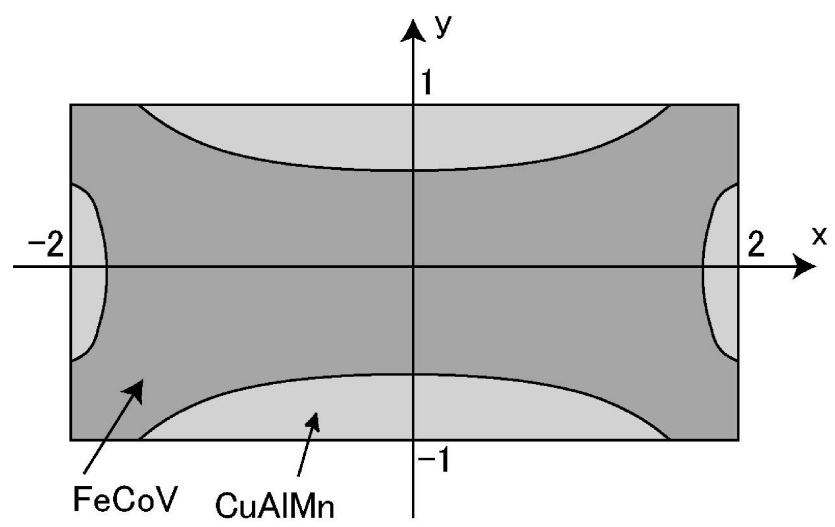

Figure 11. Optimized rectangular section of the composite made of ferromagnetic, FeCoV and superelastic SMA, CUAIMn.

If $\mathrm{FeCoV}\left(G_{\mathrm{f}}=70 \mathrm{GPa}, \tau_{\mathrm{f}}=231 \mathrm{MPa}\right)$ is used as a ferromagnetic material, and CuAlMn is used as a superelastic SMA, then for $\omega=0.003, a=2$, and $b=1, \gamma / \omega a<0.55$ is obtained from the requirement of $G_{\mathrm{f}} \gamma<\tau_{\mathrm{f}}$. Figure 11 shows the optimized rectangular section of the composite obtained by this design, where the dark area of FeCoV satisfies $\gamma /$ $\omega a<0.55$.

Next, we examine the relation between the twist angle per unit length $\omega$ and the torque $T$ of the composite wire with rectangular section. The torque is calculated by

$$
\begin{aligned}
T=M_{z}=P R \cos \alpha & =\int_{-b}^{b} \int_{-a}^{a}\left(x \tau_{z y}-y \tau_{z x}\right) d x d y \\
& =\int_{-b}^{b} \int_{-a}^{a} G\left(x \gamma_{z y}-y \gamma_{z x}\right) d x d y
\end{aligned}
$$

Here, we can define three domains in the composite during loading.

Domain 1 Domain of ferromagnetic material

Domain 2 Domain with effective shear stress less than the forward transformation shear stress of SMA, $\tau_{0}$

Domain 3 Transformation domain of SMA

The effective stress in the ferromagnetic material is obtained by multiplying shear modulus $G_{\mathrm{f}}$ by the corresponding effective shear strain for the elastic deformation. In SMA, it is necessary to judge if the effective shear stress $\tau$ is below the forward transformation shear stress $\tau_{0} . \tau$ is obtained by multiplying shear modulus $G_{\mathrm{SMA}}$ by $\gamma$ if the effective shear stress of domain 2 is below the forward transformation shear stress $\tau_{0}$. In domain 3 where the effective shear stress $\tau$ reaches the upper transformation shear stress $\tau_{0}$, then $\tau=\tau_{0}$.

Because a shear strain component proportionally increases with an increase in $\omega$, by multiplying the corresponding shear strain component by the modified shear modulus $G=\tau_{0} / \gamma$, the shear stress component for which $\tau$ becomes $\tau_{0}$ is calculated. That is, Equation (21) is applicable to domain 3 by using Equation (24). Then, torque $T$ 
corresponding to the twist angle per unit length $\omega$ is calculated by Equation (21) by using the modified shear modulus in each domain according to the following equations.

Domain 1:

$$
\begin{aligned}
& \gamma<\frac{\tau_{\mathrm{f}} \omega}{G_{\mathrm{f}} \omega_{\mathrm{f}}} \\
& G=G_{\mathrm{f}}
\end{aligned}
$$

Domain 2:

$$
\begin{aligned}
& \gamma \geq \frac{\tau_{\mathrm{f}} \omega}{G_{\mathrm{f}} \omega_{f}} \quad \text { and } \quad \gamma<\frac{\tau_{0}}{G_{\mathrm{SMA}}} \\
& G=G_{\mathrm{SMA}}
\end{aligned}
$$

Domain 3:

$$
\begin{aligned}
\gamma & \geq \frac{\tau_{\mathrm{f}} \omega}{G_{\mathrm{f}} \omega_{\mathrm{f}}} \quad \text { and } \quad \gamma \geq \frac{\tau_{0}}{G_{\mathrm{SMA}}} \\
G & =\frac{\tau_{0}}{\gamma}
\end{aligned}
$$

where, $\omega_{\mathrm{f}}$ is input data, and it is the maximum twist angle per unit length when the cross section is optimized, $\omega_{\mathrm{f}}=0.0015$ for cross section shape of Figure 11.

Next, the case of unloading is considered. The stress in each domain decreases during the unloading, but the superelasticity in SMA was generated in Domain 3 where the effective shear stress reached the transformation stress during the preceding loading, it is necessary to divide Domain 3 into three sub-domains.

Domain 3-1 $\tau$ above the reverse transformation stress $\tau_{1}$

Domain 3-2 $\tau$ equal to the reverse transformation stress $\tau_{1}$

Domain 3-3 $\tau$ below the reverse transformation stress $\tau_{1}$

For Domain 3-1, the effective shear stress $\tau$ is larger than the reverse transformation stress $\tau_{1}$. The shear stress component of $\tau=\tau_{0}$ is calculated by multiplying modified shear modulus of Equation (24) by the shear strain component, and it decreases from this stress state elastically in proportion to $G_{\mathrm{SMA}}$ in Domain 3. That is, the shear stress component is calculated by multiplying the modified shear modulus of Equation (26) by the shear strain component in the range of the effective shear strain of Equation (25).

Domain 3-1

$$
\begin{gathered}
\gamma>\frac{\tau_{1}}{G_{\mathrm{SMA}}} \quad \text { and } \quad \gamma \leq \frac{\left(\tau_{0}-\tau_{1}\right) \omega}{G_{\mathrm{SMA}}\left(\omega_{\mathrm{f}}-\omega\right)} \\
G=\frac{\tau_{0}}{\gamma}-G_{\mathrm{SMA}}
\end{gathered}
$$

For Domain 3-2, because the effective shear stress reaches the reverse transformation stress $\tau_{1}$, the shear stress remains constant, i.e. $\tau=\tau_{1}$. That is, the shear stress is calculated 
by multiplying the modified shear modulus of Equation (28) by the shear strain in the range of effective shear strain of Equation (27).

Domain 3-2

$$
\begin{gathered}
\gamma>\frac{\tau_{1}}{G_{\mathrm{SMA}}} \text { and } \gamma>\frac{\left(\tau_{0}-\tau_{1}\right) \omega}{G_{\mathrm{SMA}}\left(\omega_{\mathrm{f}}-\omega\right)} \\
G=\frac{\tau_{1}}{\gamma}
\end{gathered}
$$

For Domain 3-3, the superelasticity disappears because the effective shear stress lowers more than $\tau_{1}$. The range of effective shear strain and modified shear modulus are given by

Domain 3-3

$$
\begin{aligned}
\gamma & \leq \frac{\tau_{1}}{G_{\text {SMA }}} \\
G & =G_{\text {SMA }}
\end{aligned}
$$

The torque $T$ corresponding to $\omega$ can be analyzed from Equation (21) by calculating effective shear strain of each area using the modified shear modulus corresponding to each domain defined by Equations (22)-(24), (26), (28) and (30). The relation between the force and displacement of a spring can be calculated by using Equations (15) and (16).

\section{Analytical Results and Discussion}

Based on the above model, we made predictions of the torque $(T)$ - twist angle $(\omega)$ relation, and also of the spring force $(P)$ - displacement $(\delta)$ relation where the idealized stress-strain relations of ferromagnetic $\mathrm{FeCoV}$ and superelastic $\mathrm{CuAlMn}$ shown in Figure 12 are used.

Figure 13 shows the analytical results for the case of maximum twist angle per unit length $\omega=0.003$ of a composite plate wire with $a=2 \mathrm{~mm}$ (width is $4 \mathrm{~mm}$ ), and $b=1 \mathrm{~mm}$ (height is $2 \mathrm{~mm}$ ). Figure 13(a) shows the relation between the torque and the normalized

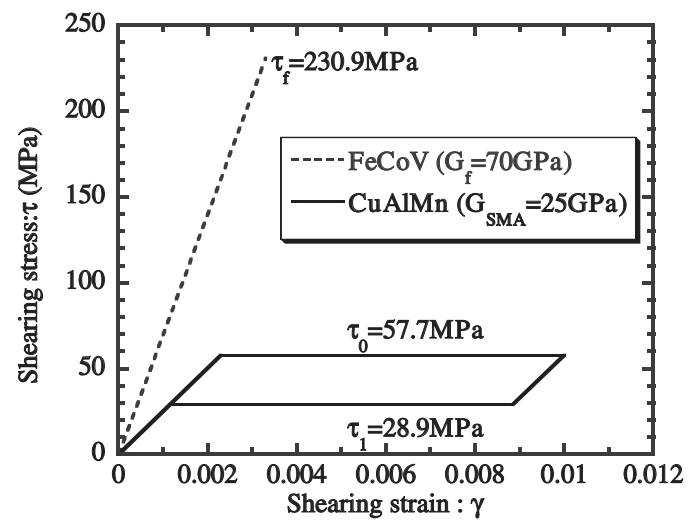

Figure 12. Idealized stress-strain curves of FeCoV and CuAIMn. 

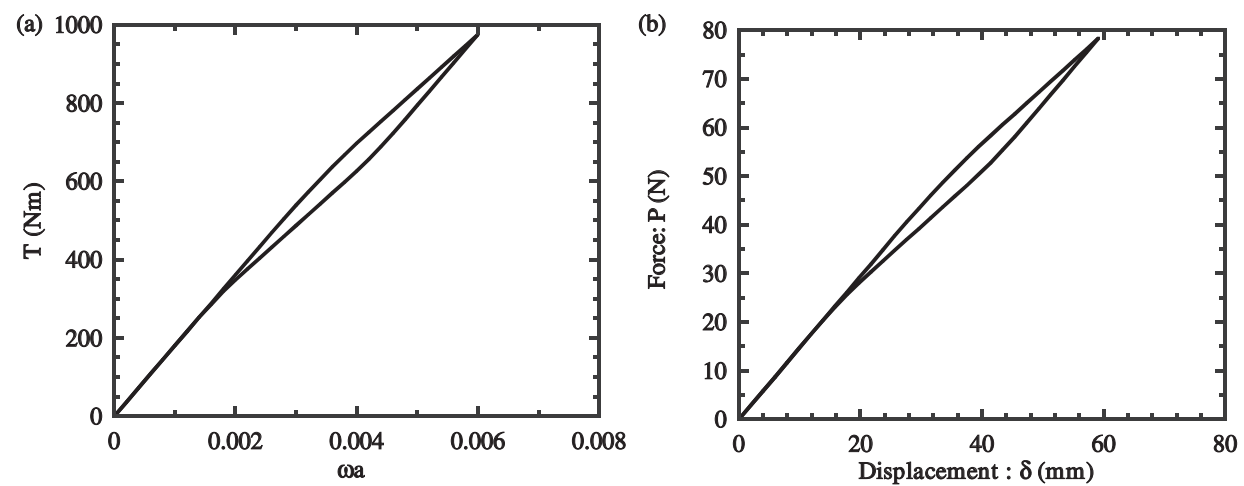

Figure 13. Superelastic behavior of Fe/CuAIMn composite spring: (a) relation between torque and normalized twist angle; (b) spring force $(P)$-displacement $(\delta)$ curve.

twist angle, indicating that the torque rises proportionally as the twist angle increases, and the transformation of SMA begins at $\omega a=0.0025$, reaching the transformation stress with $\omega a=0.0042$ in all domain of SMA. After $\omega a$ reaches 0.006 , the superelastic loop exhibits the reverse transformation corresponding to the unloading.

Figure 13(b) shows the relation between the spring force and the displacement of the coil spring of length $L=100 \mathrm{~mm}$, diameter $D=25 \mathrm{~mm}$, pitch $p=5 \mathrm{~mm}$ and number of turns $n=20$. The maximum displacement of this coiled spring was $59.2 \mathrm{~mm}$, the spring force became $78.4 \mathrm{~N}$.

We made a parametric study to examine the effects of each parameter on the $P-\delta$ relation. Figure 14 shows the analytical results of the $P-\delta$ relations influenced by various parameters, (a) $G_{\mathrm{SMA}}$, (b) $\tau_{0}$, (c) $G_{\mathrm{f}}$, (d) $\tau_{\mathrm{f}}$ and (e) $\tau_{1}$. From Figure $14(\mathrm{a})$, it is clear that shear modulus of superelasticity SMA does not influence the maximum displacement and the maximum spring force. It is noted from Figure 14(b), that the spring force increases with an increase in forward transformation shear stress $\tau_{0}$. It is clear from Figure 14(c), that the spring force does not change and only the maximum displacement increases if the shear modulus of the ferromagnetic material becomes small resulting in larger displacement of the spring. It can be seen from Figure 14(d), that both the spring force and displacement increase the superelastic behavior when the yield stress of the ferromagnetic material increases. It is noted from Figure 14(e), that the lower limit of superelastic loop decreases if the reverse transformation stress $\tau_{1}$ decreases.

In summary, larger $\tau_{\mathrm{f}}$ of the ferromagnetic material and softer ferromagnetic material will provide a spring actuator with larger displacement. And, to obtain large force of the spring, use of SMA of larger $\tau_{0}$ is desired.

We are examining two kinds $\left[\mathrm{Fe}\left(G_{\mathrm{f}}=70 \mathrm{GPa}, \tau_{\mathrm{f}}=116 \mathrm{MPa}\right)\right.$ and $\mathrm{FeCoV}\left(G_{\mathrm{f}}=70 \mathrm{GPa}\right.$, $\left.\left.\tau_{\mathrm{f}}=231 \mathrm{MPa}\right)\right]$ as a ferromagnetic material from the view point of low cost and easiness of processing. It follows from Figure 14(d) that Fe does not show the superelastic behavior and the spring force and displacement are small. Therefore, we considered that FeCoV whose $\tau_{\mathrm{f}}$ is large was suitable as the ferromagnetic material.

Next, we shall compare the mechanical performance ( $P-\delta$ relation) of a spring between "rectangular" and "square" cross section. To this end, the cross section area of the square is made equal to that of the rectangular studied earlier (Figure 12). The analytical results of the optimum square cross section of $\mathrm{FeCoV} / \mathrm{CuAlMn}$ composite are shown in Figure 15(a), while the $P-\delta$ relation of the FSMA spring with this square cross section is given in 


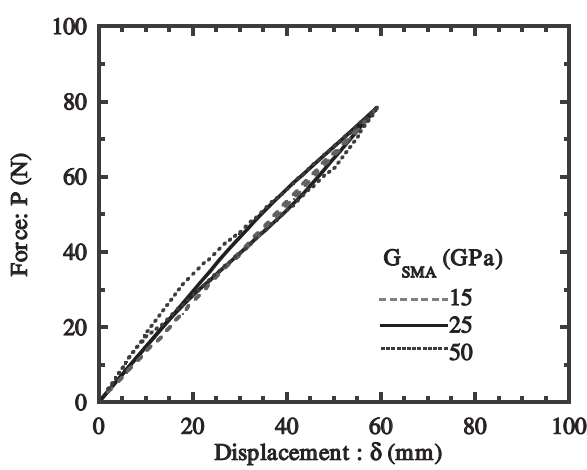

(a)

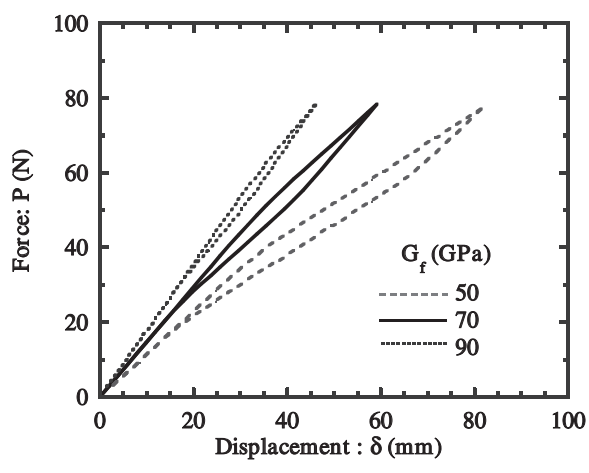

(c)

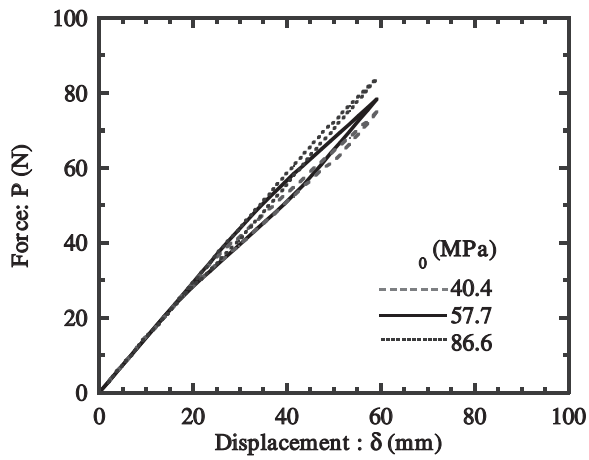

(b)



(d)

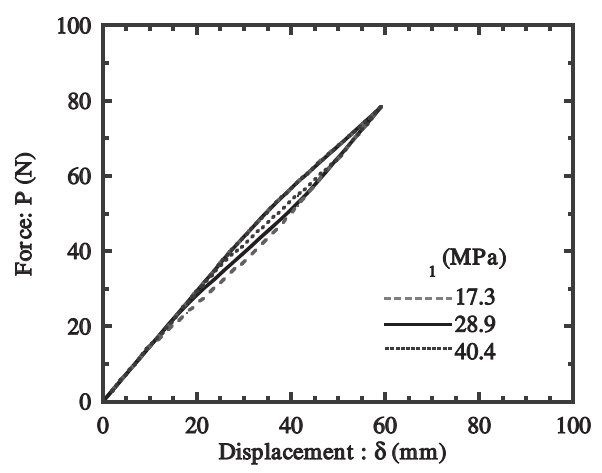

(e)

Figure 14. Effects of various parameters on $P-\delta$ relation of FMSA composite springs: (a) SMA shear modulus, $G_{S M A}$; (b) forward transformation shear stress, $\tau_{0}$; (c) shear modulus of a ferromagnetic material, $G_{f}$; (d) the yield stress in shear of a ferromagnetic material, $\tau_{f}$; and (e) reverse transformation shear stress, $\tau_{1}$.

Figure 15(b) as a dashed line where the results of the rectangular cross section are also shown by solid line. A comparison between the square cross section of Figure 15(a) and the rectangular cross section of Figure 11 reveals that the FSMA composite spring with square cross section provides larger force capability than that with the rectangular cross section for the same cross section area. However, the effectiveness of using the spring with 


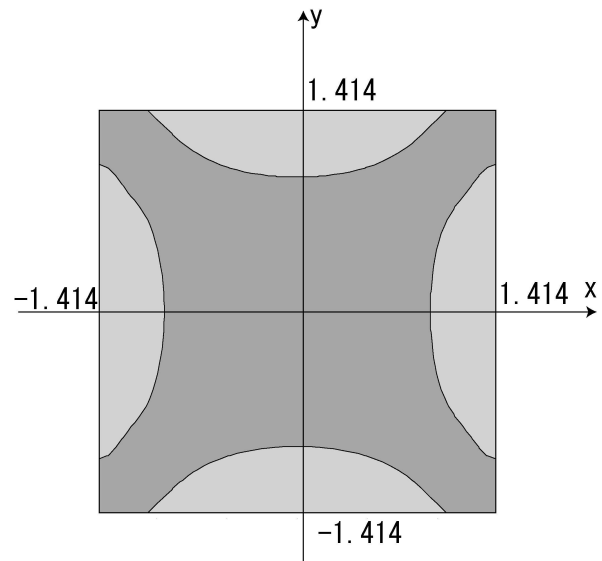

(a)

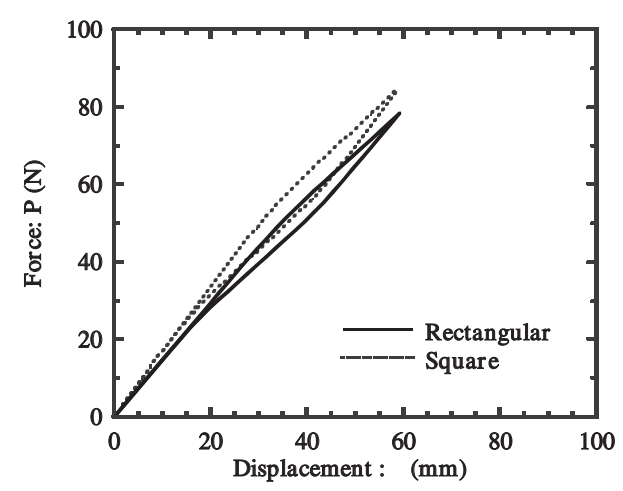

(b)

Figure 15. Superelastic behavior of Fe/CuAIMn composites: (a) shape of cross section; (b) spring forcedisplacement curve.

the square cross section remains to be determined after its effectiveness of inducing large magnetic force between the neighboring turns of the spring.

\section{CONCLUSION}

The predicted results of the bending moment-curvature of a FSMA composite plate exhibit superelastic behavior of the composite beam while those of the FSMA composite spring with rectangular cross section show also similar superelastic behavior. The above superelastic behavior is the performance required for FSMA composite actuators with high force and displacement capability. The results of the simple model were used effectively for optimization of the cross section geometry of two types of FSMA composite, bending and torsion types.

\section{ACKNOWLEDGMENT}

This study was supported by a Grant from AFOSR to University of Washington (F49620-02-1-0028) where Dr. Les Lee is the Program Manager.

\section{APPENDIX}

\section{Relation Between Bending Moment and Curvature}

The relation between the normalized bending moment and the normalized curvature of the FMSA composite plate is classified into the following eight patterns as shown Figure A.1. 


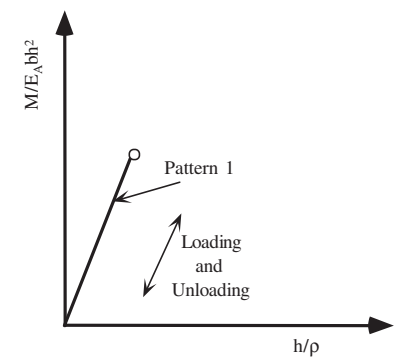

(a)



(b)

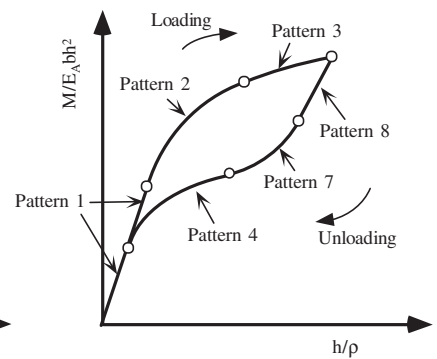

(c)

Figure A1. Relation between normalized bending moment and normalized curvature: (a) Case 1; (b) Case 2; (c) Case 3.

Case 1 is constructed with only Pattern 1. (Figure A.1(a))

Case 2 is constructed with Patterns 1 and 2 for the loading, and Patterns 1, 4, 5, and 6 for the unloading. (Figure A.1(b))

Case 3 is constructed with Patterns 1, 2 and 3 for the loading, and Patterns 1, 4, 7, and 8 for the unloading. (Figure A.1(c))

Equations of each pattern are shown as follows.

Pattern 1 (Cases 1-3)

$$
\frac{M}{E_{\mathrm{SMA}} b h^{2}}=\frac{h}{\rho}\left[\frac{E_{\mathrm{f}}}{E_{\mathrm{SMA}}}\left\{\frac{1}{3}\left(\frac{h_{\mathrm{f}}}{h}\right)^{3}-\frac{1}{2} \frac{\xi_{1}}{h}\left(\frac{h_{\mathrm{f}}}{h}\right)^{2}\right\}+\frac{1}{3}\left\{1-\left(\frac{h_{\mathrm{f}}}{h}\right)^{3}\right\}-\frac{1}{2} \frac{\xi_{1}}{h}\left\{1-\left(\frac{h_{\mathrm{f}}}{h}\right)^{2}\right\}\right]
$$

where, $\xi_{1}$ is the distance of the neutral axis.

$$
\frac{\xi_{1}}{h}=\frac{\left(\left(E_{\mathrm{f}} / E_{\mathrm{SMA}}\right)-1\right)\left(h_{\mathrm{f}} / h\right)^{2}+1}{2\left\{\left(\left(E_{\mathrm{f}} / E_{\mathrm{SMA}}\right)-1\right)\left(h_{\mathrm{f}} / h\right)+1\right\}}
$$

Pattern 2 (Cases 2,3)

$$
\begin{aligned}
\frac{M}{E_{\mathrm{SMA}} b h^{2}}=\frac{h}{\rho}\left[\frac{E_{\mathrm{f}}}{E_{\mathrm{SMA}}}\left\{\frac{1}{3}\left(\frac{h_{\mathrm{f}}}{h}\right)^{3}-\frac{1}{2} \frac{\xi_{2}}{h}\left(\frac{h_{\mathrm{f}}}{h}\right)^{2}\right\}+\frac{1}{3}\left\{\left(\frac{y_{1}}{h}\right)^{3}-\left(\frac{h_{\mathrm{f}}}{h}\right)^{3}\right\}-\frac{1}{2} \frac{\xi_{2}}{h}\left\{\left(\frac{y_{1}}{h}\right)^{2}-\left(\frac{h_{\mathrm{f}}}{h}\right)^{2}\right\}\right] \\
+\frac{1}{2} \frac{\sigma_{0}}{E_{\mathrm{SMA}}}\left\{1-\left(\frac{y_{1}}{h}\right)^{2}\right\}
\end{aligned}
$$

where, $\xi_{2}$ is the distance of the neutral axis, and $y_{1}$ is the position for $\sigma=\sigma_{0}$.

$$
\begin{aligned}
\frac{\xi_{2}}{h}= & -\left\{\left(\frac{E_{\mathrm{f}}}{E_{\mathrm{SMA}}}-1\right) \frac{h_{\mathrm{f}}}{h}+\frac{\sigma_{0}}{E_{\mathrm{SMA}}} \frac{\rho}{h}\right\} \\
& +\sqrt{\frac{E_{\mathrm{f}}}{E_{\mathrm{SMA}}}\left(\frac{E_{\mathrm{f}}}{E_{\mathrm{SMA}}}-1\right)\left(\frac{h_{\mathrm{f}}}{h}\right)^{2}+2 \frac{\sigma_{0}}{E_{\mathrm{SMA}}} \frac{\rho}{h}\left\{1+\left(\frac{E_{\mathrm{f}}}{E_{\mathrm{SMA}}}-1\right) \frac{h_{\mathrm{f}}}{h}\right\}} \\
\frac{y_{1}}{h}= & \frac{\xi_{2}}{h}+\frac{\sigma_{0}}{E_{\mathrm{SMA}}} \frac{\rho}{h}
\end{aligned}
$$


Pattern 3 (Case 3)

$$
\frac{M}{E_{\mathrm{SMA}} b h^{2}}=\frac{h}{\rho} \frac{E_{\mathrm{f}}}{E_{\mathrm{SMA}}}\left\{\frac{1}{3}\left(\frac{h_{\mathrm{f}}}{h}\right)^{3}-\frac{1}{2} \frac{\xi_{3}}{h}\left(\frac{h_{\mathrm{f}}}{h}\right)^{2}\right\}+\frac{1}{2} \frac{\sigma_{0}}{E_{\mathrm{SMA}}}\left\{1-\left(\frac{h_{\mathrm{f}}}{h}\right)^{2}\right\}
$$

where, $\xi_{3}$ is the distance of the neutral axis.

$$
\frac{\xi_{3}}{h}=\frac{\sigma_{0}}{E_{\mathrm{f}}} \frac{\rho}{h}\left(\frac{h}{h_{\mathrm{f}}}-1\right)+\frac{1}{2} \frac{h_{\mathrm{f}}}{h}
$$

Pattern 4 (Cases 2,3)

$$
\begin{gathered}
\frac{M}{E_{\mathrm{SMA}} b h^{2}}=\frac{h}{\rho}\left[\frac{E_{\mathrm{f}}}{E_{\mathrm{SMA}}}\left\{\frac{1}{3}\left(\frac{h_{\mathrm{f}}}{h}\right)^{3}-\frac{1}{2} \frac{\xi_{4}}{h}\left(\frac{h_{\mathrm{f}}}{h}\right)^{2}\right\}+\frac{1}{3}\left\{\left(\frac{y_{2}}{h}\right)^{3}-\left(\frac{h_{\mathrm{f}}}{h}\right)^{3}\right\}-\frac{1}{2} \frac{\xi_{4}}{h}\left\{\left(\frac{y_{1}}{h}\right)^{2}-\left(\frac{h_{\mathrm{f}}}{h}\right)^{2}\right\}\right] \\
+\frac{1}{2} \frac{\sigma_{1}}{E_{\mathrm{SMA}}}\left\{1-\left(\frac{y_{2}}{h}\right)^{2}\right\}
\end{gathered}
$$

where, $\xi_{4}$ is the distance of the neutral axis, and $y_{2}$ is the position for $\sigma=\sigma_{0}$.

$$
\begin{aligned}
\frac{\xi_{4}}{h}= & -\left\{\left(\frac{E_{\mathrm{f}}}{E_{\mathrm{SMA}}}-1\right) \frac{h_{\mathrm{f}}}{h}+\frac{\sigma_{1}}{E_{\mathrm{SMA}}} \frac{\rho}{h}\right\} \\
& +\sqrt{\frac{E_{\mathrm{f}}}{E_{\mathrm{SMA}}}\left(\frac{E_{\mathrm{f}}}{E_{\mathrm{SMA}}}-1\right)\left(\frac{h_{\mathrm{f}}}{h}\right)^{2}+2 \frac{\sigma_{1}}{E_{\mathrm{SMA}}} \frac{\rho}{h}\left\{1+\left(\frac{E_{\mathrm{f}}}{E_{\mathrm{SMA}}}-1\right) \frac{h_{\mathrm{f}}}{h}\right\}} \\
\frac{y_{2}}{h}= & \frac{\xi_{4}}{h}+\frac{\sigma_{1}}{E_{\mathrm{SMA}}} \frac{\rho}{h}
\end{aligned}
$$

Pattern 5 (Case 2)

$$
\begin{aligned}
\frac{M}{E_{\mathrm{SMA}} b h^{2}}= & \frac{h}{\rho}\left[\frac{1}{3}\left\{\left(\frac{E_{\mathrm{f}}}{E_{\mathrm{SMA}}}-1\right)\left(\frac{h_{\mathrm{f}}}{h}\right)^{3}+\left(\frac{y_{3}}{h}\right)^{3}\right\}-\frac{1}{2} \frac{\xi_{5}}{h}\left\{\left(\frac{E_{\mathrm{f}}}{E_{\mathrm{SMA}}}-1\right)\left(\frac{h_{\mathrm{f}}}{h}\right)^{2}+\left(\frac{y_{3}}{h}\right)^{2}\right\}\right] \\
& -\frac{h}{\rho_{1}}\left[\frac{1}{3}\left\{\left(\frac{y_{3}}{h}\right)^{3}-\left(\frac{Y_{1}}{h}\right)^{3}\right\}-\frac{1}{2} \frac{\xi_{5}}{h}\left\{\left(\frac{y_{3}}{h}\right)^{2}-\left(\frac{Y_{1}}{h}\right)^{2}\right\}\right]+\frac{1}{2} \frac{\sigma_{0}}{E_{\mathrm{SMA}}}\left\{\left(\frac{y_{3}}{h}\right)^{2}-\left(\frac{Y_{1}}{h}\right)^{2}\right\} \\
& +\frac{1}{2} \frac{\sigma_{1}}{E_{\mathrm{SMA}}}\left\{1-\left(\frac{y_{3}}{h}\right)^{2}\right\}
\end{aligned}
$$

where, $\xi_{5}$ is the distance of the neutral axis. 


$$
\begin{aligned}
\frac{\xi_{5}}{h}= & \frac{-B_{3}+\sqrt{B_{3}^{2}-A_{3} C_{3}}}{A_{3}} \\
A_{3}= & 1-\frac{h}{\rho_{1}} \frac{\rho}{h} \\
B_{3}= & \left(\frac{E_{\mathrm{f}}}{E_{\mathrm{SMA}}}-1\right) \frac{h_{\mathrm{f}}}{h}+\left(\frac{h}{\rho_{1}} \frac{Y_{1}}{h}-\frac{\sigma_{0}-\sigma_{1}}{E_{\mathrm{SMA}}}\right) \frac{\rho}{h} \\
C_{3}= & -\left(\frac{E_{\mathrm{f}}}{E_{\mathrm{SMA}}}-1\right)\left(\frac{h_{\mathrm{f}}}{h}\right)^{2}-\left\{\left(\frac{h}{\rho_{1}} \frac{Y_{1}}{h}-2 \frac{\sigma_{0}}{E_{\mathrm{SMA}}}\right) \frac{Y_{1}}{h}+2 \frac{\sigma_{1}}{E_{\mathrm{SMA}}}-\left(\frac{\sigma_{0}-\sigma_{1}}{E_{\mathrm{SMA}}}\right)^{2} \frac{\rho_{1}}{\rho_{1}-\rho} \frac{\rho}{h}\right\} \frac{\rho}{h} \\
\frac{y_{3}}{h}= & \frac{\xi_{5}}{h}-\frac{\sigma_{0}-\sigma_{1}}{E_{\mathrm{SMA}}} \frac{\rho_{1}}{\rho_{1}-\rho} \frac{\rho}{h} \\
\frac{Y_{1}}{h}= & \left(\frac{\sigma_{\mathrm{f}}}{E_{\mathrm{f}}}+\frac{\sigma_{0}}{E_{\mathrm{SMA}}}\right) \frac{\rho_{1}}{h} \\
\frac{h}{\rho_{1}}= & \left\{\left(\frac{\sigma_{\mathrm{f}}}{E_{\mathrm{f}}}+\frac{\sigma_{0}}{E_{\mathrm{SMA}}}\right)^{2}\right\} /\left\{-\left\{\frac{\sigma_{\mathrm{f}}}{E_{\mathrm{f}}}\left(\frac{E_{\mathrm{f}}}{E_{\mathrm{SMA}}}-1\right) \frac{h_{\mathrm{f}}}{h}-\frac{\sigma_{0}}{E_{\mathrm{SMA}}}\right\}\right. \\
& +\sqrt{\left.\left\{\frac{\sigma_{\mathrm{f}}}{E_{\mathrm{f}}}\left(\frac{E_{\mathrm{f}}}{E_{\mathrm{SMA}}}-1\right) \frac{h_{\mathrm{f}}}{h}-\frac{\sigma_{0}}{E_{\mathrm{SMA}}}\right\}^{2}+\left(\frac{\sigma_{\mathrm{f}}}{E_{\mathrm{f}}}+\frac{\sigma_{0}}{E_{\mathrm{SMA}}}\right)^{2}\left(\frac{E_{\mathrm{f}}}{E_{\mathrm{SMA}}}-1\right)\left(\frac{h_{\mathrm{f}}}{h}\right)^{2}\right\}}
\end{aligned}
$$

Pattern 6 (Case 2)

$$
\begin{aligned}
\frac{M}{E_{\mathrm{SMA}} b h^{2}}= & \frac{h}{\rho}\left[\frac{E_{\mathrm{f}}}{E_{\mathrm{SMA}}}\left\{\frac{1}{3}\left(\frac{h_{\mathrm{f}}}{h}\right)^{3}-\frac{1}{2} \frac{\xi_{6}}{h}\left(\frac{h_{\mathrm{f}}}{h}\right)^{2}\right\}+\frac{1}{3}\left\{1-\left(\frac{h_{\mathrm{f}}}{h}\right)^{3}\right\}-\frac{1}{2} \frac{\xi_{6}}{h}\left\{1-\left(\frac{h_{\mathrm{f}}}{h}\right)^{2}\right\}\right] \\
& -\frac{h}{\rho_{1}}\left[\frac{1}{3}\left\{1-\left(\frac{Y_{1}}{h}\right)^{3}\right\}-\frac{1}{2} \frac{\xi_{6}}{h}\left\{1-\left(\frac{Y_{1}}{h}\right)^{2}\right\}\right]+\frac{1}{2} \frac{\sigma_{0}}{E_{\mathrm{SMA}}}\left\{1-\left(\frac{Y_{1}}{h}\right)^{2}\right\}
\end{aligned}
$$

where, $\xi_{6}$ is the distance of the neutral axis.

$$
\frac{\xi_{6}}{h}=\frac{\left(\left(E_{\mathrm{f}} / E_{\mathrm{SMA}}\right)-1\right)\left(h_{\mathrm{f}} / h\right)^{2}+1-\left\{\left(h / \rho_{1}\right)\left(1+\left(Y_{1} / h\right)\right)-2\left(\sigma_{0} / E_{\mathrm{SMA}}\right)\right\}\left(1-\left(Y_{1} / h\right)\right)(\rho / h)}{2\left\{\left(\left(E_{\mathrm{f}} / E_{\mathrm{SMA}}\right)-1\right)\left(h_{\mathrm{f}} / h\right)+1-\left(h / \rho_{1}\right)\left(1-\left(Y_{1} / h\right)\right)(\rho / h)\right\}}
$$

Pattern 7 (Case 3)

$$
\begin{aligned}
\frac{M}{E_{\mathrm{SMA}} b h^{2}}=\frac{h}{\rho}[ & \left.\frac{1}{3}\left\{\left(\frac{E_{\mathrm{f}}}{E_{\mathrm{SMA}}}-1\right)\left(\frac{h_{\mathrm{f}}}{h}\right)^{3}+\left(\frac{y_{4}}{h}\right)^{3}\right\}-\frac{1}{2} \frac{\xi_{7}}{h}\left\{\left(\frac{E_{\mathrm{f}}}{E_{\mathrm{SMA}}}-1\right)\left(\frac{h_{\mathrm{f}}}{h}\right)^{2}+\left(\frac{y_{4}}{h}\right)^{2}\right\}\right] \\
& -\frac{h}{\rho_{2}}\left[\frac{1}{3}\left\{\left(\frac{y_{4}}{h}\right)^{3}-\left(\frac{h_{\mathrm{f}}}{h}\right)^{3}\right\}-\frac{1}{2} \frac{\xi_{7}}{h}\left\{\left(\frac{y_{4}}{h}\right)^{2}-\left(\frac{h_{\mathrm{f}}}{h}\right)^{2}\right\}\right]+\frac{1}{2} \frac{\sigma_{0}}{E_{\mathrm{SMA}}}\left\{\left(\frac{y_{4}}{h}\right)^{2}-\left(\frac{h_{\mathrm{f}}}{h}\right)^{2}\right\} \\
& +\frac{1}{2} \frac{\sigma_{1}}{E_{\mathrm{SMA}}}\left\{1-\left(\frac{y_{4}}{h}\right)^{2}\right\}
\end{aligned}
$$


where, $\xi_{7}$ is the distance of the neutral axis.

$$
\begin{aligned}
\frac{\xi_{7}}{h} & =\frac{-B_{4}+\sqrt{B_{4}^{2}-A_{4} C_{4}}}{A_{4}} \\
A_{4} & =1-\frac{h}{\rho_{2}} \frac{\rho}{h} \\
B_{4} & =\left(\frac{E_{\mathrm{f}}}{E_{\mathrm{SMA}}}-1\right) \frac{h_{\mathrm{f}}}{h}+\left(\frac{h}{\rho_{2}} \frac{h_{\mathrm{f}}}{h}-\frac{\sigma_{0}-\sigma_{1}}{E_{\mathrm{SMA}}}\right) \frac{\rho}{h} \\
C_{4} & =-\left(\frac{E_{\mathrm{f}}}{E_{\mathrm{SMA}}}-1\right)\left(\frac{h_{\mathrm{f}}}{h}\right)^{2}-\left\{\left(\frac{h}{\rho_{2}} \frac{h_{\mathrm{f}}}{h}-2 \frac{\sigma_{0}}{E_{\mathrm{SMA}}}\right) \frac{h_{\mathrm{f}}}{h}+2 \frac{\sigma_{1}}{E_{\mathrm{SMA}}}-\left(\frac{\sigma_{0}-\sigma_{1}}{E_{\mathrm{SMA}}}\right)^{2} \frac{\rho_{2}}{\rho_{2}-\rho} \frac{\rho}{h}\right\} \frac{\rho}{h} \\
\frac{y_{4}}{h} & =\frac{\xi_{7}}{h}-\frac{\sigma_{0}-\sigma_{1}}{E_{\mathrm{SMA}}} \frac{\rho_{2}}{\rho_{2}-\rho} \frac{\rho}{h} \\
\frac{h}{\rho_{2}} & =2 \frac{h}{h_{\mathrm{f}}}\left\{\frac{\sigma_{\mathrm{f}}}{E_{\mathrm{f}}}-\frac{\sigma_{0}}{E_{\mathrm{f}}}\left(\frac{h}{h_{\mathrm{f}}}-1\right)\right\}
\end{aligned}
$$

Pattern 8 (Case 3)

$$
\begin{aligned}
\frac{M}{E_{\mathrm{SMA}} b h^{2}}=\frac{h}{\rho} & {\left[\frac{E_{\mathrm{f}}}{E_{\mathrm{SMA}}}\left\{\frac{1}{3}\left(\frac{h_{\mathrm{f}}}{h}\right)^{3}-\frac{1}{2} \frac{\xi_{8}}{h}\left(\frac{h_{\mathrm{f}}}{h}\right)^{2}\right\}+\frac{1}{3}\left\{1-\left(\frac{h_{\mathrm{f}}}{h}\right)^{3}\right\}-\frac{1}{2} \frac{\xi_{8}}{h}\left\{1-\left(\frac{h_{\mathrm{f}}}{h}\right)^{2}\right\}\right] } \\
& -\frac{h}{\rho_{2}}\left[\frac{1}{3}\left\{1-\left(\frac{h_{\mathrm{f}}}{h}\right)^{3}\right\}-\frac{1}{2} \frac{\xi_{8}}{h}\left\{1-\left(\frac{h_{\mathrm{f}}}{h}\right)^{2}\right\}\right]+\frac{1}{2} \frac{\sigma_{0}}{E_{\mathrm{SMA}}}\left\{1-\left(\frac{h_{\mathrm{f}}}{h}\right)^{2}\right\}
\end{aligned}
$$

where, $\xi_{8}$ is the distance of the neutral axis.

$$
\frac{\xi_{8}}{h}=\frac{\left(\left(E_{\mathrm{f}} / E_{\mathrm{SMA}}\right)-1\right)\left(h_{\mathrm{f}} / h\right)^{2}+1-\left\{\left(h / \rho_{2}\right)\left(1+\left(h_{\mathrm{f}} / h\right)\right)-2\left(\sigma_{0} / E_{\mathrm{SMA}}\right)\right\}\left(1-\left(h_{\mathrm{f}} / h\right)\right)(\rho / h)}{2\left\{\left(\left(E_{\mathrm{f}} / E_{\mathrm{SMA}}\right)-1\right)\left(h_{\mathrm{f}} / h\right)+1-\left(h / \rho_{2}\right)\left(1-\left(h_{\mathrm{f}} / h\right)\right)(\rho / h)\right\}}
$$

\section{USEFUL RANGE}

The useful range of the curvature of each pattern is shown as follows.

\section{Case 1}

Pattern 1 (Loading and Unloading)

$$
0 \leq \frac{h}{\rho} \leq \frac{\sigma_{\mathrm{f}}}{E_{\mathrm{f}}} \frac{2\left\{1+\left(\left(E_{\mathrm{f}} / E_{\mathrm{SMA}}\right)-1\right)\left(h_{\mathrm{f}} / h\right)\right\}}{1+\left(\left(E_{\mathrm{f}} / E_{\mathrm{SMA}}\right)-1\right)\left(h_{\mathrm{f}} / h\right)^{2}}
$$




\section{Case 2}

Pattern 1 (Loading)

$$
0 \leq \frac{h}{\rho} \leq \frac{\sigma_{0}}{E_{\mathrm{SMA}}} \frac{2\left\{1+\left(\left(E_{\mathrm{f}} / E_{\mathrm{SMA}}\right)-1\right)\left(h_{\mathrm{f}} / h\right)\right\}}{1+\left(\left(E_{\mathrm{f}} / E_{\mathrm{SMA}}\right)-1\right)\left(2-\left(h_{\mathrm{f}} / h\right)\right)\left(h_{\mathrm{f}} / h\right)}
$$

Pattern 2 (Loading)

$$
\frac{\sigma_{0}}{E_{\mathrm{SMA}}} \frac{2\left\{1+\left(\left(E_{\mathrm{f}} / E_{\mathrm{SMA}}\right)-1\right)\left(h_{\mathrm{f}} / h\right)\right\}}{1+\left(\left(E_{\mathrm{f}} / E_{\mathrm{SMA}}\right)-1\right)\left(2-\left(h_{\mathrm{f}} / h\right)\right)\left(h_{\mathrm{f}} / h\right)}<\frac{h}{\rho} \leq \frac{h}{\rho_{1}}
$$

Pattern 1 (Unloading)

$$
0 \leq \frac{h}{\rho} \leq \frac{\sigma_{1}}{E_{\mathrm{SMA}}} \frac{2\left\{1+\left(\left(E_{\mathrm{f}} / E_{\mathrm{SMA}}\right)-1\right)\left(h_{\mathrm{f}} / h\right)\right\}}{1+\left(\left(E_{\mathrm{f}} / E_{\mathrm{SMA}}\right)-1\right)\left(2-\left(h_{\mathrm{f}} / h\right)\right)\left(h_{\mathrm{f}} / h\right)}
$$

Pattern 4 (Unloading)

$$
\begin{aligned}
\frac{\sigma_{1}}{E_{\mathrm{SMA}}} \frac{2\left\{1+\left(\left(E_{\mathrm{f}} / E_{\mathrm{SMA}}\right)-1\right)\left(h_{\mathrm{f}} / h\right)\right\}}{1+\left(\left(E_{\mathrm{f}} / E_{\mathrm{SMA}}\right)-1\right)\left(2-\left(h_{\mathrm{f}} / h\right)\right)\left(h_{\mathrm{f}} / h\right)} \\
\quad<\frac{h}{\rho} \leq \frac{2\left(\sigma_{1} / E_{\mathrm{SMA}}\right)\left\{1+\left(\left(E_{\mathrm{f}} / E_{\mathrm{SMA}}\right)-1\right)\left(h_{\mathrm{f}} / h\right)\right\}}{\left(Y_{1} / h\right)^{2}+\left(\left(E_{\mathrm{f}} / E_{\mathrm{SMA}}\right)-1\right)\left(2\left(Y_{1} / h\right)-\left(h_{\mathrm{f}} / h\right)\right)\left(h_{\mathrm{f}} / h\right)}
\end{aligned}
$$

Pattern 5 (Unloading)

$$
\frac{2\left(\sigma_{1} / E_{\mathrm{SMA}}\right)\left\{1+\left(\left(E_{\mathrm{f}} / E_{\mathrm{SMA}}\right)-1\right)\left(h_{\mathrm{f}} / h\right)\right\}}{\left(Y_{1} / h\right)^{2}+\left(\left(E_{\mathrm{f}} / E_{\mathrm{SMA}}\right)-1\right)\left(2\left(Y / h_{1}\right)-\left(h_{\mathrm{f}} / h\right)\right)\left(h_{\mathrm{f}} / h\right)}<\frac{h}{\rho} \leq \frac{2 A_{1}}{-B_{1}-\sqrt{B_{1}^{2}-4 A_{1} C_{1}}}
$$

where,

$$
\begin{aligned}
A_{1}= & \left\{2 \frac{\sigma_{1}}{E_{\mathrm{SMA}}}+\left(1-\frac{Y_{1}}{h}\right) \frac{h}{\rho_{1}}\right\}\left(1-\frac{Y_{1}}{h}\right) \\
B_{1}= & 2\left\{\left(\frac{E_{\mathrm{f}}}{E_{\mathrm{SMA}}}-1\right) \frac{h_{\mathrm{f}}}{h}+1\right\}\left(\frac{\sigma_{0}-\sigma_{1}}{E_{\mathrm{SMA}}} \frac{\rho_{1}}{h}-1\right)+\left(\frac{E_{\mathrm{f}}}{E_{\mathrm{SMA}}}-1\right)\left(\frac{h_{\mathrm{f}}}{h}\right)^{2} \\
& +1-\left(1-\frac{Y_{1}}{h}\right)\left(1-\frac{Y_{1}}{h}+2 \frac{\sigma_{0}}{E_{\mathrm{SMA}}} \frac{\rho_{1}}{h}\right) \\
C_{1}= & \frac{\rho_{1}}{h}\left\{\left(\frac{E_{\mathrm{f}}}{E_{\mathrm{SMA}}}-1\right)\left(2-\frac{h_{\mathrm{f}}}{h}\right) \frac{h_{\mathrm{f}}}{h}+1\right\}
\end{aligned}
$$


Pattern 6 (Unloading)

$$
\frac{2 A_{1}}{-B_{1}-\sqrt{B_{1}^{2}-4 A_{1} C_{1}}}<\frac{h}{\rho} \leq \frac{h}{\rho_{1}}
$$

\section{Case 3}

Pattern 1 (Loading)

$$
0 \leq \frac{h}{\rho} \leq\left(\sigma_{0} / E_{\mathrm{SMA}}\right) \frac{2\left\{1+\left(\left(E_{\mathrm{f}} / E_{\mathrm{SMA}}\right)-1\right)\left(h_{\mathrm{f}} / h\right)\right\}}{1+\left(\left(E_{\mathrm{f}} / E_{\mathrm{SMA}}\right)-1\right)\left(2-\left(h_{\mathrm{f}} / h\right)\right)\left(h_{\mathrm{f}} / h\right)}
$$

Pattern 2 (Loading)

$$
\frac{\sigma_{0}}{E_{\mathrm{SMA}}} \frac{2\left\{1+\left(\left(E_{\mathrm{f}} / E_{\mathrm{SMA}}\right)-1\right)\left(h_{\mathrm{f}} / h\right)\right\}}{1+\left(\left(E_{\mathrm{f}} / E_{\mathrm{SMA}}\right)-1\right)\left(2-\left(h_{\mathrm{f}} / h\right)\right)\left(h_{\mathrm{f}} / h\right)}<\frac{h}{\rho} \leq 2 \frac{h}{h_{\mathrm{f}}}\left\{\frac{\sigma_{0}}{E_{\mathrm{SMA}}}+\frac{\sigma_{0}}{E_{\mathrm{f}}}\left(\frac{h_{\mathrm{f}}}{h}-1\right)\right\}
$$

Pattern 3 (Loading)

$$
2 \frac{h}{h_{\mathrm{f}}}\left\{\frac{\sigma_{0}}{E_{\mathrm{SMA}}}+\frac{\sigma_{0}}{E_{\mathrm{f}}}\left(\frac{h_{\mathrm{f}}}{h}-1\right)\right\}<\frac{h}{\rho} \leq \frac{h}{\rho_{2}}
$$

Pattern 1 (Unloading)

$$
0 \leq \frac{h}{\rho} \leq \frac{\sigma_{1}}{E_{\mathrm{SMA}}} \frac{2\left\{1+\left(\left(E_{\mathrm{f}} / E_{\mathrm{SMA}}\right)-1\right)\left(h_{\mathrm{f}} / h\right)\right\}}{1+\left(\left(E_{\mathrm{f}} / E_{\mathrm{SMA}}\right)-1\right)\left(2-\left(h_{\mathrm{f}} / h\right)\right)\left(h_{\mathrm{f}} / h\right)}
$$

Pattern 4 (Unloading)

$$
\begin{aligned}
& \frac{\sigma_{1}}{E_{\mathrm{SMA}}} \frac{2\left\{1+\left(\left(E_{\mathrm{f}} / E_{\mathrm{SMA}}\right)-1\right)\left(h_{\mathrm{f}} / h\right)\right\}}{1+\left(\left(E_{\mathrm{f}} / E_{\mathrm{SMA}}\right)-1\right)\left(2-\left(h_{\mathrm{f}} / h\right)\right)\left(h_{\mathrm{f}} / h\right)} \\
& <\frac{h}{\rho} \leq \frac{2\left(\sigma_{1} / E_{\mathrm{SMA}}\right)\left\{1+\left(\left(E_{\mathrm{f}} / E_{\mathrm{SMA}}\right)-1\right)\left(h_{\mathrm{f}} / h\right)\right\}}{\left(Y_{1} / h\right)^{2}+\left(\left(E_{\mathrm{f}} / E_{\mathrm{SMA}}\right)-1\right)\left(2\left(Y_{1} / h\right)-\left(h_{\mathrm{f}} / h\right)\right)\left(h_{\mathrm{f}} / h\right)}
\end{aligned}
$$

Pattern 7 (Unloading)

$$
\frac{2\left(\sigma_{1} / E_{\mathrm{SMA}}\right)\left\{1+\left(\left(E_{\mathrm{f}} / E_{\mathrm{SMA}}\right)-1\right)\left(h_{\mathrm{f}} / h\right)\right\}}{\left(Y_{1} / h\right)^{2}+\left(\left(E_{\mathrm{f}} / E_{\mathrm{SMA}}\right)-1\right)\left(2\left(Y_{1} / h\right)-\left(h_{\mathrm{f}} / h\right)\right)\left(h_{\mathrm{f}} / h\right)}<\frac{h}{\rho} \leq \frac{2 A_{2}}{-B_{2}-\sqrt{B_{2}^{2}-4 A_{2} C_{2}}}
$$


where,

$$
\begin{aligned}
A_{2}= & \left\{2 \frac{\sigma_{1}}{E_{\mathrm{SMA}}}+\left(1-\frac{h_{\mathrm{f}}}{h}\right) \frac{h}{\rho_{2}}\right\}\left(1-\frac{h_{\mathrm{f}}}{h}\right) \\
B_{2}= & 2\left\{\left(\frac{E_{\mathrm{f}}}{E_{\mathrm{SMA}}}-1\right) \frac{h_{\mathrm{f}}}{h}+1\right\}\left(\frac{\sigma_{0}-\sigma_{1}}{E_{\mathrm{SMA}}} \frac{\rho_{2}}{h}-1\right)+\left(\frac{E_{\mathrm{f}}}{E_{\mathrm{SMA}}}-1\right)\left(\frac{h_{\mathrm{f}}}{h}\right)^{2} \\
& +1-\left(1-\frac{h_{\mathrm{f}}}{h}\right)\left(1-\frac{h_{\mathrm{f}}}{h}+2 \frac{\sigma_{0}}{E_{\mathrm{SMA}}} \frac{\rho_{2}}{h}\right) \\
C_{2}= & \frac{\rho_{2}}{h}\left\{\left(\frac{E_{\mathrm{f}}}{E_{\mathrm{SMA}}}-1\right)\left(2-\frac{h_{\mathrm{f}}}{h}\right) \frac{h_{\mathrm{f}}}{h}+1\right\}
\end{aligned}
$$

Pattern 8 (Unloading)

$$
\frac{2 A_{2}}{-B_{2}-\sqrt{B_{2}^{2}-4 A_{2} C_{2}}}<\frac{h}{\rho} \leq \frac{h}{\rho_{2}}
$$

\section{REFERENCES}

1. Kato, H., Wada, T., Tagawa, T., Liang, Y. and Taya, M. (2001). Proc. of 50th Anniversary of Japan Society of Materials Sciences, A: 296-305.

2. Kato, H., Liang, Y., Wada, T., Tagawa, T., Taya, M. and Mori, T. (2002). Meter. Sci. Eng. A, A332: 134-139.

3. Birman, V. (1997). Int. J. Mechanical Sciences, 39(10): 1139-1149.

4. Lee, J.J. and Choi, S. (1999). Composite Structures, 47(1-4): 695-703.

5. Thompson, S.P. and Loughlan, J. (2000). Thin-Walled Structures, 36(4): 231-263.

6. Marfia, S. and Sacco, E. (2003). AIAA Journal, 41(1): 100-109.

7. Auricchio, F., Taylor, R.L. and Lubliner, J. (1997). Comput. Methods Appl. Mech. Engrg., 146: 281-312.

8. Matsunaga, Y. and Taya, M. (2002). In: Chang, F.K. (ed.), Proc. 10th US-Japan Conference on Composite Materials, pp. 40-50, Sept. 16-18, DES Tech Pub. Lancaster, PA, Keynote Paper.

9. Taya, M., Wada, T., Kusaka, M. and Lee, R.C.C. (2003). Proc. SPIE, In: Anderson, E. (ed.), Vol. 5054, pp. 156-164, Industrial and Commercial Applications of Smart Structures and Technologies.

10. Wada, T., Lee, R.C.C., Chen, S.H.H., Kusaka, M. and Taya, M. (2003). In: Anderson, E. (ed.), Proc. SPIE, Vol. 5054, pp. 125-134, Industrial and Commercial Applications of Smart structures and Technologies.

11. Timoshenko, S.P. and Goodier, J.N. Theory of Elasticity, 3rd edn., McGraw. 
